\title{
Dubois torsion, $A$-polynomial and quantum invariants
}

\author{
Charles D. Frohman and Joanna Kania-Bartoszynska ${ }^{1}$
}

\begin{abstract}
It is shown that for knots with a sufficiently regular character variety the Dubois torsion detects the $A$-polynomial of the knot. A global formula for the integral of the Dubois torsion is given. The formula looks like the heat kernel regularization of the formula for the Witten-Reshetikhin-Turaev invariant of the double of the knot complement. The Dubois torsion is recognized as the pushforward of a measure on the character variety of the double of the knot complement coming from the square root of Reidemeister torsion. This is used to motivate a conjecture about quantum invariants detecting the $A$-polynomial.
\end{abstract}

Mathematics Subject Classification (2010). 57M.

Keywords. Reidemeister torsion, Turaev-Viro invariants, Laplace's method.

\section{Introduction}

There is a close connection between the colored Jones polynomial and the $\mathrm{SL}_{2}(\mathbb{C})$ characters of knot groups. The first intimation of this connection appeared in Bullock's work on the skein modules of knot complements [5]. The idea was developed more formally in [6] and [39]. The point is that the Kauffman bracket skein module of a three manifold $M$, when the complex parameter is set to be equal to -1 , is the coordinate ring of the unreduced scheme of the $\mathrm{SL}_{2}(\mathbb{C})$-characters of the fundamental group of $M$. The connection with the $A$-polynomial of Cooper, Culler, Gillet, Long, and Shalen [10] was first developed in [21] and [22]. The idea was to first realize the $A$-polynomial as generating the kernel of a map between rings of characters, and then deform the rings in a canonical way so that the kernel can be understood as a submodule of a skein module, called the $A$-ideal. Garoufalidis and Le developed a more algebraic setting for these ideas, that allowed them to prove the nontriviality of the noncommutative $A$-ideal, along with making many compelling conjectures [25].

It has long been conjectured that the asymptotics of the colored Jones polynomials of a knot are modulated by the $\mathrm{SL}_{2}(\mathbb{C})$ or $\mathrm{SU}(2)$-characters of the knot group.

\footnotetext{
${ }^{1}$ This material is based upon work supported by and while serving at the National Science Foundation. Any opinion, findings, and conclusions or recommendations expressed in this material are those of the authors and do not necessarily reflect the views of the National Science Foundation.
} 
As $\mathrm{SL}_{2}(\mathbb{C})$ is the complexification of $\mathrm{SU}(2)$, there is a common ground for trying to understand the relations between the representation theory of knot groups and the values of the colored Jones polynomial.

There is a conjectured asymptotic formula for the Witten-Reshetikhin-Turaev invariants for links in 3-manifolds; see [50], [20], [27], [28], [29], and [43]. It is presented here as it appeared in [38], where it was stated only for the empty link in a closed oriented three manifold $M$ :

$$
\begin{aligned}
& Z_{r}^{\mathrm{SU}(2)}(M) \\
& \quad \sim_{r \rightarrow \infty} e^{-3 \pi \mathbf{i}\left(1+b^{1}(M)\right) / 4} \sum_{[A]} e^{2 \pi \mathbf{i} \operatorname{CS}(A)} r^{\left(h_{A}^{1}-h_{A}^{0}\right) / 2} e^{-2 \pi \mathbf{i}\left(I_{A} / 4+h_{A}^{0} / 8\right)} \tau_{M}(A)^{1 / 2} .
\end{aligned}
$$

Here $b^{1}(M)$ is the first Betti number of $M, \operatorname{CS}(A)$ is the Chern-Simons invariant of the flat $\mathrm{SU}(2)$ connection $A$, and $[A]$ denotes the gauge equivalence class of $A$. Next, $h_{A}^{i}$ is the rank of the $i$-th cohomology of $M$ with coefficients in the su(2)-bundle twisted by the adjoint action of the monodromy of $A$, and $I_{A}$ is the spectral flow of the signature operator along a path connecting $A$ to the trivial flat connection. Finally, $\tau_{M}(A)$ is the Reidemeister torsion of the $\operatorname{Ad} A$-twisted chain complex $C^{i}(M, \operatorname{Ad} A)$.

When the $\mathrm{SU}(2)$-character variety has positive dimension, the sum in the above formula can be interpreted as an integral with respect to an appropriate volume form. In this case, if we restrict to connections that are irreducible, the square root of the Reidemeister torsion defines a natural measure on the character variety. In the case of torus knots, Kashaev and Dubois have worked out the asymptotics of the colored Jones polynomial and found in [16] an asymptotic expansion having the same flavor as formula (1).

In this paper we explore the Reidemeister torsion of Dubois [13] as a measure on the regular part of the SU(2)-character variety of a knot complement. Exploiting the connection with the Kauffman bracket skein algebra of the torus [21], we use it to define a seminorm on that algebra. When the SU(2)-character variety is sufficiently nonsingular the radical of this seminorm is the ideal of functions that vanish on the image of the irreducible representations of the knot complement in the character variety of the torus.

We go on to develop a global formula for the seminorm that looks like the WittenReshetikhin-Turaev invariant of the skein in the double of the knot complement. This leads us to a conjectural characterization of the $A$-polynomial of knots with sufficiently regular character varieties in terms of quantum invariants.

Rozansky [43] first recognized that for regular representations of 3-manifolds, the square root of the Reidemeister torsion defines an invariant volume form on the regular part of the character variety. As part of the motivation of our conjecture we prove that the Dubois torsion of a knot is a geometrically motivated evaluation of the square root of the Reidemeister torsion of the double of the knot complement. 
The plan of the paper is as follows. In Section 2 we talk about the representation spaces of knot groups. In Section 3 we review the Dubois torsion and describe the corresponding seminorm. In Section 4 we prove that the Dubois torsion of a knot complement is a geometrically motivated evaluation of the square root of the Reidemeister torsion of its double. In Section 5 we give a global computation of the seminorm. Finally, in Section 6 we compare the answer from the global computation to the formula for the Witten-Reshetikhin-Turaev invariants of links in the double of the knot complement. Throughout the paper we work the example of the trefoil knot to make the exposition concrete.

The authors thank Thomas Kerler who helped us understand the global computation, and Paul Kirk who helped with the interpretation of the conjectured asymptotic formula.

\section{Representations of knot groups}

In this section we recall the definition of the A-ideal of a knot and illustrate it with the computation for the trefoil.

2.1. The $\boldsymbol{A}$-ideal. Let $K \subset S^{3}$ be a knot, and $N(K)$ an open regular neighborhood of $K$ so that $S^{3}-N(K)$ is a smooth manifold with boundary a torus $T^{2}$. The fundamental group of the torus is $\mathbb{Z} \times \mathbb{Z}$ with generators the longitude $\lambda$ and meridian $\mu$. We are interested in the image of the representations of the fundamental group of $S^{3}-N(K)$ in the representations of the fundamental group of $T^{2}$ under restriction. Although the study of this image was initiated using $\operatorname{SL}(2, \mathbb{C})$ representations [10], in this paper we study SU(2) representations. In [10] the authors also pass to a cover, so that the image is cut out by a single polynomial, which they dubbed the $A$-polynomial. It is common to throw out the component of the abelian representations or just focus on the component that contains the holonomy of the complete hyperbolic structure and still refer to the polynomial cutting out that variety as the $A$-polynomial.

Recall that $\mathrm{SU}(2)$ consists of two by two complex matrices of the form

$$
\left(\begin{array}{cc}
\alpha & \beta \\
-\bar{\beta} & \bar{\alpha}
\end{array}\right)
$$

where $(\alpha, \beta) \in \mathbb{C}^{2}$ satisfies $|\alpha|^{2}+|\beta|^{2}=1$. Alternatively, you can think of $\operatorname{SU}(2)$ as the unit quaternions. In the quaternionic model, each element of SU(2) can be written as $\cos \varphi+\sin \varphi \vec{P}$, where $\vec{P}$ is a unit vector in $\mathbb{R}^{3}$. This second model yields an elegant way of working with the tangent space of $\mathrm{SU}(2)$ at the identity, that is,

$$
\operatorname{su}(2)=\mathrm{T}_{1} S^{3}=\mathbb{R}^{3} .
$$

Under this identification, the adjoint representation Ad: $\mathrm{SU}(2) \rightarrow \operatorname{End}(\operatorname{su}(2))$,

$$
\operatorname{Ad}_{X}(\vec{v})=X \vec{v} X^{-1}
$$


is easily understood. If $X=\cos \varphi+\sin \varphi \vec{P}$ then $\operatorname{Ad}_{X}$ acts as a rotation with angle $2 \varphi$ radians about the axis $\vec{P}$. This simple picture led to the beautiful computations of SU(2) representations of knot groups in [9] and [33]. To simplify notation we denote the adjoint action of $X$ on $\operatorname{su}(2)$ by a lower dot, $\operatorname{Ad}_{X}(\vec{v})=X . \vec{v}$. The Lie bracket in $\operatorname{su}(2)$ is twice the cross product. Given a representation of a group $\Gamma$, $\rho: \Gamma \rightarrow \mathrm{SU}(2)$, denote by $\operatorname{Ad} \rho$ the representation into endomorphisms of su(2) obtained by following $\rho$ with the adjoint action.

If $M$ is a manifold with finitely generated fundamental group, denote the space of representations of $\pi_{1}(M)$ into SU(2) by $R(M)$. The space $R(M)$ is realized as a subset of the Cartesian product SU $(2)^{k}$, where the coordinates are the values of the representation on a finite set of generators of $\pi_{1}(M)$. Those tuples that satisfy the equations coming from the group relators exactly correspond to $R(M)$, so that not only is $R(M)$ a topological space with the subspace topology coming from $\mathrm{SU}(2)^{k}$, it is also a real algebraic variety. Actually, more is true: by interpreting the group relators as matrix equations, and then seeing each coefficient of the matrices as a real equation, the ideal generated by requiring the value of each relator to be the identity is an invariant of $M$, see [34]. The radical of that ideal is more commonly studied as an invariant.

The group SU(2) acts on $R(M)$ by conjugation. We denote the quotient space by $X(M)$. It inherits the quotient topology from $R(M)$, and is a real algebraic set, called the character variety. The respective coordinate rings are denoted by $C[R(M)]$ and $C[X(M)]$, and should be thought of as the rings of polynomial functions on the sets $R(M)$ and $X(M)$, with real coefficients. The ring $C[X(M)]$ can be identified with a quotient of the subring $C[R(M)]^{\mathrm{SU}(2)}$ of $C[R(M)]$, that is fixed under the action of $\mathrm{SU}(2)$, by the ideal of nilpotents. The ring $C[X(M)]$ is closely related to the Kauffman bracket skein module of $M$, although not necessarily equal to it. In [6], [7], and [39] the correspondence was constructed between the Kauffman bracket skein module of $M$ evaluated at a parameter -1 and the coordinate ring of the unreduced affine scheme of the $\mathrm{SL}_{2}(\mathbb{C})$ characters of the fundamental group of the manifold $M$. In this paper we are working with the SU(2) characters and with the reduced coordinate ring over the reals. When $M$ is a surface the $\mathrm{SU}(2)$ characters are a totally real submanifold of the $\mathrm{SL}_{2}(\mathbb{C})$ characters. Hence the tensor product of $C[X(M)]$ with $\mathbb{C}$ is the coordinate ring of the $\mathrm{SL}_{2}(\mathbb{C})$ character variety that contains the $\mathrm{SU}(2)$ characters. In the case of knot complements, for some knots the ring $C[X(M)]$ might not contain the information about all the $\mathrm{SL}_{2}(\mathbb{C})$ characters, so it might be a further quotient of the Kauffman bracket skein module.

Any representation $\rho: \pi_{1}\left(T^{2}\right) \rightarrow \mathrm{SU}(2)$ is determined by the values on the longitude and meridian, $\rho(\lambda)$ and $\rho(\mu)$. Since $\lambda$ and $\mu$ commute, the matrices $\rho(\lambda)$ and $\rho(\mu)$ are simultaneously diagonalizable. Thus they are conjugate to a pair

$$
\left(\left(\begin{array}{cc}
l & 0 \\
0 & l^{-1}
\end{array}\right),\left(\begin{array}{cc}
m & 0 \\
0 & m^{-1}
\end{array}\right)\right)
$$

where $l, m \in S^{1} \subset \mathbb{C}$. These coordinates are ambiguous, since the two matrices can 
be simultaneously conjugated by $\left(\begin{array}{cc}0 & -1 \\ 1 & 0\end{array}\right)$ with the result of exchanging the positions of the eigenvalues $l$ and $l^{-1}$, and $m$ and $m^{-1}$. From this we see that the conjugacy classes of SU(2)-representations of $\pi_{1}\left(T^{2}\right)$ can be identified with $S^{1} \times S^{1} / \sim$, where the equivalence relation $\sim$ comes from the hyperelliptic involution

$$
\theta: S^{1} \times S^{1} \longrightarrow S^{1} \times S^{1},
$$

given by $\theta(l, m)=\left(l^{-1}, m^{-1}\right)$. We have characterized $X\left(T^{2}\right)$ as a space that is called the pillowcase since it can be represented as the result of identifying two squares along their boundaries, as depicted in Figure 1.

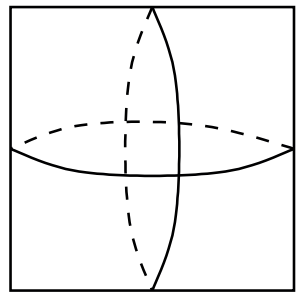

Figure 1. The pillowcase.

The coordinate ring of the pillowcase, $C\left[X\left(T^{2}\right)\right]$, can be understood as the subring of $\mathbb{R}\left[l, l^{-1}, m, m^{-1}\right]$ that is fixed by the endomorphism $\theta$. It can be shown that the functions of the form $l^{p} m^{q}+l^{-p} m^{-q}$ form a basis for $C\left[X\left(T^{2}\right)\right]$, see [21].

The inclusion map $i: T^{2} \longrightarrow S^{3}-N(K)$ induces restriction maps

$$
R\left(S^{3}-N(K)\right) \rightarrow R\left(T^{2}\right)
$$

and

$$
\psi: X\left(S^{3}-N(K)\right) \longrightarrow X\left(T^{2}\right) .
$$

We are especially interested in the ring homomorphism induced by $\psi$,

$$
\Psi: C\left[X\left(T^{2}\right)\right] \longrightarrow C\left[X\left(S^{3}-N(K)\right)\right] .
$$

By definition, if $f \in C\left[X\left(T^{2}\right)\right]$, then $\Psi(f)=f \circ \psi$. The radical of the kernel of $\Psi$ is an ideal $B(K) \subset C\left[X\left(T^{2}\right)\right]$ which we call the $B$-ideal of the knot $K$. It is the ideal of polynomial functions on $X\left(T^{2}\right)$ that are zero on the image of the restriction map $\psi$. We are less interested in the characters of the abelian representations. The ideal of functions that vanish on the image of the irreducible characters is denoted by $A(K)$.

2.2. An example. The complement of the trefoil collapses onto a $\mathrm{CW}$-complex with one vertex $v$, two edges $x$ and $y$, and a single two cell whose attaching map is $x^{2} y^{-3}$, see Figure 2. 


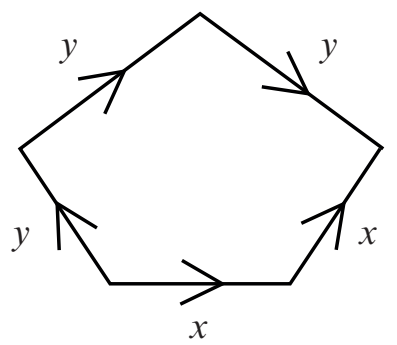

Figure 2. The CW-complex for the trefoil.

This can be visualized by seeing the trefoil as lying on an unknotted torus. In this setting there is a two complex consisting of the two cores of the handlebodies bounded by the torus, and a singular annulus that runs twice around one core and three times around the other, and misses the trefoil. Pinch the singular annulus along a crosscut. The pinch point is the vertex, the remnants of the cores are the two edges, and the result of pinching the annulus is the two-cell.

This two-complex gives rise to a presentation of the fundamental group of the knot complement. There is one generator for each edge and one relator for the twocell. It is important to be able to express the meridian and longitude in terms of the generators. The complement of the trefoil knot has the fundamental group

$$
\pi_{1}\left(S^{3}-N(K)\right)=\left\langle x, y \mid x^{2}=y^{3}\right\rangle .
$$

The meridian is given by $\mu=x y^{-1}$, and the longitude is $\lambda=x^{2} \mu^{-6}=x^{2}\left(x y^{-1}\right)^{-6}$. The meridian and longitude commute, hence the word $\lambda \mu \lambda^{-1} \mu^{-1}$ in the free group on $x$ and $y$ is in the normal closure of the relator $r=x^{-2} y^{3}$. Following Dubois [14],

$$
\lambda \mu \lambda^{-1} \mu^{-1}=x^{2} \mu^{-6} \mu \mu^{6} x^{-2} \mu^{-1}=x r x^{-1} \mu r^{-1} \mu^{-1} .
$$

From this equation, we can build a map of the torus $T^{2}$ into the two-complex of the knot complement which is homotopic to the inclusion of the boundary torus. Fill in a square with sides from the commutator by two 2-cells corresponding to the word above. This gives a map of a disk into the two-complex, so that points on opposite sides of the square get mapped to the same point. The map descends to a map of a torus into the two-complex. This torus represents the boundary of the knot exterior.

The representations of $\pi_{1}\left(S^{3}-N(K)\right)$ into $\mathrm{SU}(2)$ can be identified with a subset of $\mathrm{SU}(2) \times \mathrm{SU}(2)$. Give $\mathrm{SU}(2) \times \mathrm{SU}(2)$ coordinates $(X, Y)$ and send

$$
\rho: \pi_{1}\left(S^{3}-N(K)\right) \longrightarrow \mathrm{SU}(2)
$$

to $(\rho(x), \rho(y))$. To correspond to a representation, a pair $(X, Y)$ must satisfy the equation $X^{2}=Y^{3}$. There are two components of this subset of $\mathrm{SU}(2) \times \mathrm{SU}(2)$ : the points coming from abelian representations, and the points coming from irreducible representations. 
From [33] we know that if $\rho: \pi_{1}\left(S^{3}-N(K)\right) \rightarrow \mathrm{SU}(2)$ is irreducible then $\rho\left(x^{2}\right)=\rho\left(y^{3}\right)=-1$. This leads to an easy parametrization of the components of $R\left(S^{3}-N(K)\right)$. To parametrize the abelian representations let

$$
\alpha: \mathrm{SU}(2) \longrightarrow \mathrm{SU}(2) \times \mathrm{SU}(2)
$$

be given by $\alpha(Z)=\left(Z^{3}, Z^{2}\right)$. To parametrize the irreducible representations, let

$$
\beta: \mathrm{SU}(2) \times(0, \pi) \longrightarrow \mathrm{SU}(2) \times \mathrm{SU}(2)
$$

be given by

$$
\beta(A, t)=\left(A \mathbf{i} A^{-1}, A\left(\cos \frac{\pi}{3}+\sin \frac{\pi}{3}(\cos t \mathbf{i}+\sin t \mathbf{j})\right) A^{-1}\right) .
$$

The parametrization $\alpha$ of the abelian representations is one to one. The parametrization $\beta$ of the irreducible representations is 2 to 1 , because the center of $\mathrm{SU}(2)$ is in the kernel of the map coming from conjugation. Notice that the abelian representations have codimension 3 in $\mathrm{SU}(2) \times \mathrm{SU}(2)$ and the irreducible representations have codimension 2 .

The map $\beta$ extends smoothly to

$$
\bar{\beta}: \mathrm{SU}(2) \times[0, \pi] \longrightarrow \mathrm{SU}(2) \times \mathrm{SU}(2) .
$$

The images of $\mathrm{SU}(2) \times\{0\}$ and $\mathrm{SU}(2) \times\{\pi\}$ under $\bar{\beta}$ are two spheres of abelian representations. The points in the abelian representations that lie in the closure of the irreducible representations are called bifurcation points. At the bifurcation points the squares of the eigenvalues of the matrices that are images of the meridian are roots of the Alexander polynomial of the trefoil knot. This phenomenon occurs for any knot; see [12], [24], and [33].

The image of the irreducible representations of the complement of the trefoil in the pillowcase under restriction is depicted in Figure 3.

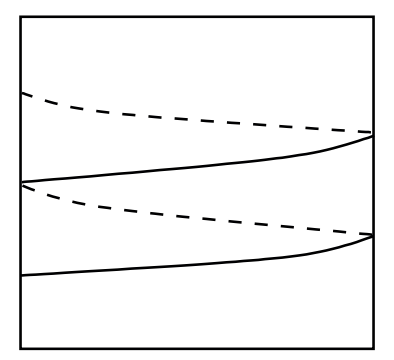

Figure 3. Image of the characters of the trefoil in the pillowcase.

The $A$-polynomial of the trefoil is given by

$$
A(l, m)=l+m^{-6},
$$


where $l$ and $m$ are the upper left hand corner of the diagonalized $\rho(\lambda)$ and $\rho(\mu)$ respectively, as in (2). The $A$-ideal of the trefoil is generated in $\mathbb{R}\left[l, l^{-1}, m, m^{-1}\right]$ by the polynomials

$$
l+l^{-1}+m^{6}+m^{-6}
$$

and

$$
l m+l^{-1} m^{-1}+m^{5}+m^{-5} .
$$

The computation can be found in [26].

\section{Dubois torsion}

This section presents Dubois torsion for knots, which in turn is used to construct a seminorm on $C\left[X\left(T^{2}\right)\right]$ whose radical is $A(K)$.

3.1. Reidemeister torsion and volume. If $\mathbf{u}=\left\{\vec{u}_{i}\right\}$ and $\mathbf{w}=\left\{\vec{w}_{j}\right\}$ are two ordered bases for the finite dimensional vector space $V$, then each $\vec{w}_{j}=\alpha_{j}^{i} \vec{u}_{i}$, where, following Einstein's summation convention, the last equation represents a sum over all $i$. The determinant of the change of basis matrix $\left(\alpha_{j}^{i}\right)$ is denoted $[\mathbf{w} / \mathbf{u}]$. Given a finite dimensional cochain complex $C=\left(C^{i}, \delta^{i+1}\right)$ whose cochain groups are vector spaces over $\mathbb{R}$ with preferred ordered basis $\mathbf{c}_{i}$ for $C^{i}$, and a collection $\mathbf{h}_{i}$ of $i$ cochains that give rise to an ordered basis for $H^{i}(C)$, choose an ordered basis $\mathbf{b}_{i}$ for the complement of the cocycles $Z^{i}(C)$. Finally let $\overline{\mathbf{b}}_{i}$ be the image of $\mathbf{b}_{i}$ under $\delta^{i+1}: C^{i} \rightarrow C^{i+1}$. Notice that the concatenation $\overline{\mathbf{b}}_{i-1}, \mathbf{h}_{i}, \mathbf{b}_{i}$ is an ordered basis for $C^{i}$. The Reidemeister torsion of the cochain complex $C$ evaluated on the basis $\mathbf{h}$ is defined by:

$$
\tau(\mathbf{h})=\left|\frac{\prod_{i \text { odd }}\left[\overline{\mathbf{b}}_{i-1}, \mathbf{h}_{i}, \mathbf{b}_{i} / \mathbf{c}_{\mathbf{i}}\right]}{\prod_{i \text { even }}\left[\overline{\mathbf{b}}_{i-1}, \mathbf{h}_{i}, \mathbf{b}_{i} / \mathbf{c}_{\mathbf{i}}\right]}\right| .
$$

In the case where the cochain groups are inner product spaces, there is a preferred class of bases. Specifically, any orthonormal basis can be used. This is because the change of basis matrix between two orthonormal bases has determinant \pm 1 . Since we are working with the absolute value, $\tau(\mathbf{h})$ does not depend on the choice of bases for $C^{i}$ as long as $\mathbf{c}_{i}$ are orthonormal.

The Reidemeister torsion transforms as a top dimensional form, i.e. a volume form, on

$$
\bigoplus_{i \text { even }} H^{i}(C) \oplus \bigoplus_{i \text { odd }}\left(H^{i}(C)\right)^{*}
$$

The definition and properties of Reidemeister torsion can be found in [37], [40], [45], [46], and [49]. The tools developed for manipulating torsion in [37] are fundamental.

This paper relies on interpreting the Reidemeister torsion of a chain complex in terms of Riemannian volume forms. To make this interpretation clear, we review some geometry of volume. Given an oriented inner product space $V$ there is a distinguished 
top dimensional form characterized by the fact that it evaluates to 1 on any positively oriented orthonormal ordered basis of $V$. We call this the volume form.

Recall that, if $L: V \rightarrow W$ is a linear map of inner product spaces, we can restrict the domain of $L$ to the perpendicular of its kernel and restrict its range to its image, $L:(\operatorname{ker} L)^{\perp} \rightarrow \operatorname{im} L$, to get an isomorphism of inner product spaces. We do not have orientations for these two spaces, so we do not know the sign of the determinant of $L$, but we can define the content of $L$, denoted by $\|L\|$, to be

$$
\|L\|=\sqrt{\operatorname{det}\left(L^{*} \circ L\right)}=\sqrt{\operatorname{det}\left(L \circ L^{*}\right)},
$$

where we have restricted $L$ as above.

A choice of vectors $\vec{v}_{1}, \ldots, \vec{v}_{n}$ of an inner product space $V$ is equivalent to defining a linear map $L: \mathbb{R}^{n} \rightarrow V$. The content of $L$ can be thought of as the $n$-dimensional content of the parallelepiped spanned by the vectors $\vec{v}_{i}$. Given a single nonzero vector $\vec{v} \in V$, let $L: \mathbb{R} \rightarrow V$ be a linear map defined by $L(1)=\vec{v}$. Notice that $\|L\|=\|\vec{v}\|$, where the second set of double bars denotes the norm of the vector $\vec{v}$. Thus even though in this paper we use the double bar notation in two different ways: for the content of a linear map as in (5), and for the norm of a vector, the value is the same regardless.

Let $M$ be an oriented Riemannian $m$-manifold. The pointwise choice of volume forms on the tangent spaces defines a smooth $m$-form on $M, v^{M}$, called the Riemannian volume form. If $N \subset M$ is a smooth oriented submanifold, then the Riemannian metric on $M$ induces a Riemannian metric on $N$ and an associated volume form $v^{N}$. The volume forms $v^{M}$ and $v^{N}$ are related as follows. At any $p \in N$ choose an orthonormal basis $n_{1}, \ldots, n_{k}$ for the orthogonal complement of $\mathrm{T}_{p} N$ in $\mathrm{T}_{p} M$ so that as oriented vector spaces,

$$
\left\langle n_{1}, \ldots, n_{k}\right\rangle \oplus \mathrm{T}_{p} N=\mathrm{T}_{p} M .
$$

The Riemannian volume form on $N$ is the interior product of $v^{M}$ with $\left\{n_{1}, \ldots, n_{k}\right\}$. That is, for any $\left(w_{1}, \ldots w_{n}\right) \in\left(\mathrm{T}_{p} N\right)^{n}$,

$$
v^{N}\left(w_{1}, \ldots, w_{n}\right)=v^{M}\left(n_{1}, \ldots, n_{k}, w_{1}, \ldots, w_{n}\right) .
$$

Suppose instead that $v_{1}, \ldots, v_{k}$ is an arbitrary basis (not necessarily orthonormal) for the orthogonal complement of $\mathrm{T}_{p} N$. Then

$$
v^{M}\left(v_{1}, \ldots, v_{k}, w_{1}, \ldots, w_{n}\right)= \pm\|L\| v^{N}\left(w_{1}, \ldots, w_{n}\right),
$$

where $L: \mathbb{R}^{k} \rightarrow \mathrm{T}_{p} M$ is the linear map that takes the standard basis for $\mathbb{R}^{k}$ to the vectors $v_{1}, \ldots, v_{k}$.

3.2. Local definition of Dubois torsion. Let $K$ be a knot and let $W \subset S^{3}-N(K)$ be a two-complex, with one vertex, embedded in $S^{3}-N(K)$ so that $S^{3}-N(K)$ is a closed regular neighborhood of $W$. Alternatively, one can think of $S^{3}-N(K)$ as 
having a handle structure with a single zero-handle, $k$ one-handles, and $k-1$ twohandles. Denote the vertex by $v_{1}$, the edges by $e_{i}$ and the 2-cells by $f_{j}$. There is a presentation of the fundamental group of $S^{3}-N(K)$ corresponding to this complex, with one generator $x_{i}$ for each edge $e_{i}$, and one relator $r_{j}$ for each face $f_{j}$. The relator $r_{j}$ is a word in the generators $x_{i}$ coming from the attaching map of the cell $f_{j}$. Let $C_{*}(W ; \mathbb{R})$ be the chain complex that corresponds to the homology of $W$ with basis $\left\{v_{1}, e_{i}, f_{j}\right\}$. The dual chain complex $C^{*}(W ; \mathbb{R})=\operatorname{Hom}\left(C_{*}(W), \mathbb{R}\right)$ has the dual basis $\left\{v^{1}, e^{i}, f^{j}\right\}$. For instance $e^{i}: C_{*}(W ; \mathbb{R}) \rightarrow \mathbb{R}$ is the linear map so that $e^{i}\left(e_{i}\right)=1$ and the value of $e^{i}$ applied to any other basis element is 0 . We make $C^{*}(W ; \mathbb{R})$ into an inner product space by declaring the basis $\left\{v^{1}, e^{i}, f^{j}\right\}$ to be orthonormal.

Define a map

$$
\bar{r}: \mathrm{SU}(2)^{k} \longrightarrow \mathrm{SU}(2)^{k-1}
$$

by substituting the variables $x_{i}$ in the relators $\left(r_{1}, \ldots, r_{k-1}\right)$ by the matrices $\left(X_{1}, \ldots\right.$, $\left.X_{k}\right)$. The representation variety $R\left(S^{3}-N(K)\right)$ can be identified with $\bar{r}^{-1}(\mathrm{Id}, \ldots$, Id). The group $\mathrm{SU}(2)$ acts on $\mathrm{SU}(2)^{k}$ by simultaneous conjugation of the entries,

$$
c: \mathrm{SU}(2) \times \mathrm{SU}(2)^{k} \longrightarrow \mathrm{SU}(2)^{k},
$$

i.e.

$$
c\left(A, X_{1}, \ldots, X_{k}\right)=\left(A X_{1} A^{-1}, \ldots, A X_{k} A^{-1}\right),
$$

and $R\left(S^{3}-N(K)\right)$ is invariant under that action. The quotient can be identified with $X\left(S^{3}-N(K)\right)$.

Let

$$
C^{i}(W ; \operatorname{Ad} \rho)=C^{i}(\tilde{W} ; \mathbb{R}) \otimes_{\mathbb{R}(\pi)} \operatorname{su}(2),
$$

where $\tilde{W}$ is the universal cover of $W$ and $\pi$ is the fundamental group of the knot complement. This product can be identified with

$$
C^{i}(W ; \operatorname{Ad} \rho)=C^{i}(W ; \mathbb{R}) \otimes_{\mathbb{R}} \operatorname{su}(2) .
$$

This identification is not canonical since it depends on a choice of lifts of cells. However, the ambivalence in the induced volume forms discussed below is only up to sign.

Each $C^{i}(W ; \operatorname{Ad} \rho)$ is an inner product space, where the inner product is the tensor product of the inner product on $C^{*}(W)$ with the standard dot product on $\mathbb{R}^{3}=\operatorname{su}(2)$. There is a cochain complex

$$
C^{0}(W ; \operatorname{Ad} \rho) \stackrel{\delta^{1}}{\longrightarrow} C^{1}(W ; \operatorname{Ad} \rho) \stackrel{\delta^{2}}{\longrightarrow} C^{2}(W ; \operatorname{Ad} \rho),
$$

called the tangential complex. Its boundary maps can be understood as the derivatives of the action by conjugation and the derivative of $\bar{r}$. Its cohomology is closely related to the Zariski tangent space of $X\left(S^{3}-N(K)\right)$, at the conjugacy class of the 
representation $\rho: \pi_{1}\left(S^{3}-N(K)\right) \rightarrow \mathrm{SU}(2)$, as in [48]. Call the cohomology groups of complex (8) the cohomology of $W$ with coefficients in the adjoint representation associated to $\rho$.

The boundary operators are as follows. Suppose that $\rho\left(x_{i}\right)=X_{i}$ and suppose that the relator $r_{j}$ is a word in the $x_{i}$. First,

$$
\delta^{1}: C^{0}(W ; \operatorname{Ad} \rho) \longrightarrow C^{1}(W ; \operatorname{Ad} \rho)
$$

is given by

$$
\delta^{1}\left(v^{1} \otimes \vec{w}\right)=\sum_{i} e^{i} \otimes\left(X_{i}-1\right) \cdot \vec{w}
$$

Next,

$$
\delta^{2}: \oplus e^{i} \otimes \operatorname{su}(2) \longrightarrow \oplus f^{j} \otimes \operatorname{su}(2)
$$

is given by the Fox Jacobian of $\left(r_{1}, \ldots, r_{k-1}\right)$ instantiated on $\left(X_{1}, \ldots, X_{k}\right)$, and acting by the adjoint action:

$$
\delta^{2}\left(e^{i} \otimes \vec{w}\right)=\sum_{j} f^{j} \otimes \frac{\partial r_{j}}{\partial x_{i}} \cdot \vec{w} .
$$

The Fox calculus and this chain complex are described in [11], [12], [30], and [48]. Under the standard identification of $\mathrm{T}_{X} \mathrm{SU}(2)$ with su(2) by right translation, $\delta^{1}$ is the derivative of the conjugation map $c$ at the identity (where $c$ is given by (7)), and $\delta^{2}$ is the derivative of the relator map $\bar{r}$ at the representation $\rho$ (where $\bar{r}$ is defined by (6)).

The tangential complex (8) has too much cohomology for its torsion to be interpreted geometrically. To get around that Dubois [14] extends the complex so that the torsion defines a one-form on a large portion of the character variety of the knot.

A representation $\rho: \pi_{1}\left(S^{3}-N(K)\right) \rightarrow \mathrm{SU}(2)$ is regular, if Ad $\rho$ is irreducible, $H^{1}\left(S^{3}-N(K) ; \operatorname{Ad} \rho\right)=\mathbb{R}$, and the restriction of $\rho$ to the fundamental group of the boundary torus is not central. We will denote the regular representations by $R^{i}\left(S^{3}-\right.$ $N(K)$ ). Regular representations can be defined in the same way for any 3-manifold with torus boundary. The last condition is superfluous for a knot complement since the fundamental group of $S^{3}-N(K)$ is generated by conjugates of the meridian. Hence the irreducibility of the representation implies that the meridian is not sent to an element of the center of SU(2). Therefore, $H^{0}\left(T^{2} ; \operatorname{Ad} \rho\right)=\mathbb{R}$ and it is generated by $v^{1} \otimes \vec{P}$, where the meridian is sent to $\cos \varphi+\sin \varphi \vec{P}$. Euler characteristic considerations allow us to conclude that if $\rho$ is regular then $H^{2}\left(S^{3}-N(K) ; \operatorname{Ad} \rho\right)=$ $\mathbb{R}$, and the map

$$
H^{2}\left(S^{3}-N(K) ; \operatorname{Ad} \rho\right) \longrightarrow H^{2}\left(T^{2} ; \operatorname{Ad} \rho\right),
$$

from the long exact sequence of the pair $\left(S^{3}-N(K), T^{2}\right)$ is an isomorphism.

Dubois works with an extended cochain complex

$$
C^{0}(W ; \operatorname{Ad} \rho) \stackrel{\delta^{1}}{\longrightarrow} C^{1}(W ; \operatorname{Ad} \rho) \stackrel{\delta^{2}}{\longrightarrow} C^{2}(W ; \operatorname{Ad} \rho) \stackrel{\delta^{3}}{\longrightarrow} \mathbb{R} .
$$


To define $\delta^{3}$ we need to have a firm grasp on the map (9). It is the adjoint of the inclusion map on homology with coefficients in $\operatorname{Ad} \rho$.

Since the knot complement is a regular neighborhood of the two-complex $W$, it is clear that the fundamental class of the boundary torus is homologous to a chain in which each two-cell appears twice, once with positive orientation and once with negative orientation. This chain can be computed by taking words $L$ and $M$ in the $x_{i}$ corresponding to the longitude and the meridian and then writing the word $L M L^{-1} M^{-1}$ as a product of conjugates of relators and their inverses. Each relator $r_{j}$ will appear twice: once as $s_{j} r_{j} s_{j}^{-1}$ and once as $t_{j} r_{j}^{-1} t_{j}^{-1}$. The image of $\left[T^{2}\right] \otimes \vec{P}$ in $C_{2}(W ; \operatorname{Ad} \rho)=C_{2}(W) \otimes \operatorname{su}(2)$ is $\sum_{j} f_{j} \otimes\left(\operatorname{Ad}_{\rho\left(s_{j}\right)}-\operatorname{Ad}_{\rho\left(t_{j}\right)}\right) . \vec{P}$. Denote

$$
\vec{v}_{j}=\left(\operatorname{Ad}_{\rho\left(s_{j}\right)}-\operatorname{Ad}_{\rho\left(t_{j}\right)}\right) \cdot \vec{P},
$$

and let

$$
\vec{v}=\sum_{j} f^{j} \otimes \vec{v}_{j}
$$

The map $\delta^{3}: C^{2}(W ; \operatorname{Ad} \rho) \rightarrow \mathbb{R}$ is the adjoint of the inclusion map of the boundary torus into the knot complement on homology. It is equal to the dot product with $\vec{v}$.

The complex (10) only has cohomology in dimension 1, and that cohomology at a regular representation $\rho$ is the tangent space at $[\rho]$ to the character variety of the knot complement.

Here is how to pass from a tangent vector to the character variety at $[\rho]$ to a cocycle in $C^{1}(W ; \operatorname{Ad} \rho)$. For any regular representation $\rho$ there is a neighborhood of $\rho$ in $X\left(S^{3}-N(K)\right)$ which is a smooth 1-manifold. Since the quotient map

$$
R\left(S^{3}-N(K)\right) \longrightarrow X\left(S^{3}-N(K)\right)
$$

is a submersion of smooth manifolds, in a neighborhood of that point we can choose a slice $\rho_{t}:(-\varepsilon, \varepsilon) \rightarrow R\left(S^{3}-N(K)\right)$ lifting any smooth path in $X\left(S^{3}-N(K)\right)$ on a small interval. Let $\left[\rho_{t}\right]:[-\varepsilon, \varepsilon] \rightarrow X\left(S^{3}-N(K)\right)$ be a smooth path. The cochain

$$
\sum_{i} e^{i} \otimes\left(\left.\frac{d \rho_{t}\left(x_{i}\right)}{d t}\right|_{t=0}\right) \rho_{0}\left(x_{i}^{-1}\right)
$$

is a cocycle that represents the tangent vector of the path $\left[\rho_{t}\right]$.

Since the cochain complex (10) we are working with has an inner product, there is a class of preferred bases used to compute its Reidemeister torsion (4). Let $\mathbf{c}_{i}$ be an orthonormal basis for $C^{i}(W ; \mathrm{Ad} \rho)$. Let $\mathbf{b}_{0}=\mathbf{c}_{0}$, and choose $\mathbf{b}_{1}$ as in the definition of (4). Notice that the complement of the space of 2-cycles is 1-dimensional. Thus $\mathbf{b}_{2}$ consists of a single vector, which we choose to have length 1 . Recall that the only nonzero cohomology group is $H^{1}(W ; \mathrm{Ad} \rho)$ and that it corresponds to the tangent space of the character variety at $[\rho]$. Choose a tangent vector $h \in \mathrm{T}_{[\rho]} X\left(S^{3}-N(K)\right)$. 
The absolute value of the Dubois torsion evaluated at $h$ is given by

$$
\tau_{\rho}(h)=\left|\frac{\left[\overline{\mathbf{b}}_{0}, h, \mathbf{b}_{1} / \mathbf{c}_{1}\right]\|\vec{v}\|}{\left[\overline{\mathbf{b}}_{1}, \mathbf{b}_{2} / \mathbf{c}_{2}\right]}\right|,
$$

where $\vec{v}$ is the vector defined by (12). We use the subscript to emphasize the fact that we started with a choice of a regular representation $\rho$.

Dubois works with a signed refinement of torsion, using a scheme that is discussed more generally in Turaev's book [46]. In fact, Dubois proves that the torsion defines a 1-form on the manifold which is the part of the character variety of the knot corresponding to the regular representations. As the regular part of the character variety is oriented there is a well defined notion of what the sign of the integral of the torsion should be. We can't get the sign from our approach, as it is not inherent to the Gaussian integral that we use to get the global formula in Section 5.

3.3. Example continued. We will compute the torsion at the representations of the fundamental group of the complement of the trefoil knot along the path $\rho_{t}$ given by

$$
\rho_{t}(x)=\mathbf{i}, \quad \rho_{t}(y)=\cos \frac{\pi}{3}+\sin \frac{\pi}{3}(\cos t \mathbf{i}+\sin t \mathbf{j}),
$$

where $h$ is the tangent vector of the path. We are using right translation to identify the tangent space at $A$ of SU(2) with su(2), so that the value of $h$ on the word $w$ in $x$ and $y$ is

$$
\frac{d \rho}{d t}(w) \rho_{t}(w)^{-1}
$$

We are interested specifically in the ordered pair $(h(x), h(y))$ which is

$$
\left(0, \sin \frac{\pi}{3} \cos \frac{\pi}{3}(-\sin t \mathbf{i}+\cos t \mathbf{j})+\sin ^{2} \frac{\pi}{3} \mathbf{k}\right) .
$$

Recall from Section 2.2 that the complex $W$ is spanned by one 0 -cochain, denoted here by $v^{1}$, dual to the single vertex, two 1 -cochains, denoted by $e^{1}$ and $e^{2}$, dual to the two edges, and one 2-cochain, denoted by $f^{1}$, dual to the single 2-cell.

The boundary operator

$$
\delta^{0}\left(v^{1} \otimes \vec{u}\right)=e^{1} \otimes(\mathbf{i}-1) \cdot \vec{u}+e^{2} \otimes\left(\cos \frac{\pi}{3}-1+\sin \frac{\pi}{3}(\cos t \mathbf{i}+\sin t \mathbf{j})\right) \cdot \vec{u}
$$

can be understood as follows. The coefficient of $e^{1}$ is -2 times the projection onto the perpendicular to $\mathbf{i}$. The coefficient of $e^{2}$ is the projection onto the perpendicular to $\cos t \mathbf{i}+\sin t \mathbf{j}$ followed by a counterclockwise rotation by $5 \pi / 6$ degrees, followed by a homothety of $2 \sin \pi / 6$. Luckily, this will not be that important. The next boundary operator $\delta^{1}$ is given by

$$
\delta^{1}\left(e^{1} \otimes \vec{u}+e^{2} \otimes \vec{w}\right)=f^{1} \otimes\left((1+\rho(x)) \cdot \vec{u}+\left(1+\rho(y)+\rho(y)^{2}\right) \cdot \vec{w}\right) .
$$


We use the following preferred bases for the cochain groups. To start with, $C^{0}(W ; \operatorname{Ad} \rho)$ has basis $\mathbf{c}_{\mathbf{0}}=\left\{v^{1} \otimes \mathbf{i}, v^{1} \otimes \mathbf{j}, v^{1} \otimes \mathbf{k}\right\}$. The basis for $C^{1}(W ; \operatorname{Ad} \rho)$ is $\mathbf{c}_{\mathbf{1}}=\left\{e^{1} \otimes \mathbf{i}, e^{1} \otimes \mathbf{j}, e^{1} \otimes \mathbf{k}, e^{2} \otimes \mathbf{i}, e^{2} \otimes \mathbf{j}, e^{2} \otimes \mathbf{k}\right\}$. For $C^{2}(W ; \operatorname{Ad} \rho)$ we use the basis $\mathbf{c}_{2}=\left\{f^{1} \otimes \mathbf{i}, f^{1} \otimes \mathbf{j}, f^{1} \otimes \mathbf{k}\right\}$. Finally for $\mathbb{R}$ the basis consists of 1 .

As $\rho$ is irreducible, $Z^{0}(W ; \operatorname{Ad} \rho)=0$ and we can use the basis above for $C^{0}(W ; \operatorname{Ad} \rho)$ to be $\mathbf{b}_{\mathbf{0}}$. A complement of $Z^{1}(W ; \operatorname{Ad} \rho)$ is spanned by $e^{1} \otimes \mathbf{i}, e^{2} \otimes$ $(\cos t \mathbf{i}+\sin t \mathbf{j})$ so we use this as $\mathbf{b}_{\mathbf{1}}$. A complement to $Z^{2}(W ; \operatorname{Ad} \rho)$ is given by $\mathbf{k}$ so we use this as $\mathbf{b}_{\mathbf{2}}$. The last map is onto so the complement of the cocycles in $\mathbb{R}$ is $\{0\}$.

The change of basis matrix from $\mathbf{c}_{0}$ to $\mathbf{b}_{0}$ is the identity so $\left[\mathbf{b}_{0} / \mathbf{c}_{0}\right]=1$. The change of basis matrix from $\mathbf{c}_{\mathbf{1}}$ to $\overline{\mathbf{b}_{\mathbf{0}}} h \mathbf{b}_{\mathbf{1}}$ looks like

$$
\left(\begin{array}{cccccc}
0 & 0 & 0 & 0 & 1 & 0 \\
0 & -2 & 0 & 0 & 0 & 0 \\
0 & 0 & -2 & 0 & 0 & 0 \\
-\frac{3}{2}+\frac{3}{2} \cos ^{2}(t) & * & * & -\frac{\sqrt{3}}{4} \sin t & 0 & \cos t \\
\frac{3}{2} \sin t \cos t & * & * & \frac{\sqrt{3}}{4} \cos t & 0 & \sin t \\
-\frac{\sqrt{3}}{2} \sin t & * & * & \frac{3}{4} & 0 & 0
\end{array}\right)
$$

The stars are there to emphasize that if you expand the determinant correctly, they do not enter into the computation of the determinant, which is equal $6 \sin t$.

Applying $\delta^{2}$ to $\mathbf{b}_{\mathbf{2}}$ and adjoining $\mathbf{k}$ we see that the change of basis matrix from $\mathbf{c}_{\mathbf{2}}$ to $\overline{\mathbf{b}_{2}} \mathbf{k}$ is

$$
\left(\begin{array}{ccc}
2 & 3 \cos t & 0 \\
0 & 3 \sin t & 0 \\
0 & 0 & 1
\end{array}\right),
$$

whose determinant is also $6 \sin t$.

It remains to compute $\|\vec{v}\|$ defined by (11) and (12). The value of the meridian under $\rho$ is

$$
\rho(\mu)=\rho\left(x y^{-1}\right)=\sin \frac{\pi}{3} \cos t+\cos \frac{\pi}{3} \mathbf{i}-\sin \frac{\pi}{3} \sin t \mathbf{k} .
$$

The normalized imaginary part is

$$
\vec{P}=\frac{\cos \frac{\pi}{3} \mathbf{i}-\sin \frac{\pi}{3} \sin t \mathbf{k}}{\sqrt{\cos ^{2} \frac{\pi}{3}+\sin ^{2} \frac{\pi}{3} \sin ^{2} t}} .
$$

Recall that the inclusion map of the chains on the boundary to chains on $W$ takes $\left[T^{2}\right] \otimes \vec{P}$ to

$$
f_{1} \otimes(\rho(x)-\rho(\mu)) \cdot \vec{P}
$$


where $f_{1}$ is the single 2 -cell. Thus

$$
\vec{v}=f^{1} \otimes \frac{2 \sin \frac{\pi}{3} \sin t \mathbf{k}}{\sqrt{\cos ^{2} \frac{\pi}{3}+\sin ^{2} \frac{\pi}{3} \sin ^{2} t}} .
$$

Putting it all together as in (13),

$$
\tau(h)=\frac{2 \sin \frac{\pi}{3} \sin t}{\sqrt{\cos ^{2} \frac{\pi}{3}+\sin ^{2} \frac{\pi}{3} \sin ^{2} t}} .
$$

Note that this agrees with the Proposition 5.4 in [14], after you account for differences in notation, and parametrization.

Let us interpret this computation geometrically. The determinant of matrix (14) computes the volume form on $\mathrm{su}(2) \oplus \operatorname{su}(2)=C^{1}(W, \operatorname{Ad} \rho)$ which is the Riemannian volume form on $\mathrm{T}_{(X, Y)}(\mathrm{SU}(2) \times \mathrm{SU}(2))$ at the ordered pair corresponding to the representation $\rho_{t}$. The last two columns are an orthogonal complement to the image of $\mathrm{T}_{\rho} R\left(S^{3}-N(K)\right)$ at that point. Up to sign the determinant of this matrix is computing the Riemannian volume form on the representation variety of the knot at $\rho$ inherited from its embedding in $\mathrm{SU}(2) \times \mathrm{SU}(2)$. This means that we can interpret the determinant of matrix (14) as

$$
v^{R}\left(\begin{array}{cccc}
0 & 0 & 0 & 0 \\
0 & -2 & 0 & 0 \\
0 & 0 & -2 & 0 \\
-\frac{3}{2}+\frac{3}{2} \cos ^{2}(t) & * & * & -\frac{\sqrt{3}}{4} \sin t \\
\frac{3}{2} \sin t \cos t & * & * & \frac{\sqrt{3}}{4} \cos t \\
-\frac{\sqrt{3}}{2} \sin t & * & * & \frac{3}{4}
\end{array}\right)
$$

where $v^{R}$ is the Riemannian volume form on the representation variety of the knot evaluated at the column vectors of the matrix. Further contemplation of the formula reveals that the argument of $\nu^{R}$ is the derivative of $\beta(A, t)$ at $A=\mathrm{Id}$, where $\beta$ is the parametrization given by equation (3). The value of the determinant of (14) is then equal to

$$
\left(\beta_{(\mathrm{Id}, t)}\right)^{*}\left(v^{R}\right)\left(\mathbf{i}, \mathbf{j}, \mathbf{k}, \frac{d}{d t}\right) .
$$

The formula above means that we have used $\beta$ to pull the volume form $v^{R}$ back to $\mathrm{T}_{(\mathrm{Id}, t)}(\mathrm{SU}(2) \times(0, \pi))$ and then evaluated that pulled back form on an orthonormal basis.

The first three columns of matrix (14) make up a basis for the tangent space of the fiber of the projection map from the representation variety of the knot complement to 
the character variety at $\left[\rho_{t}\right]$. If we had chosen an orthonormal basis for this space, the determinant would be computing the push-forward volume on the character variety. However, the basis we chose is not orthonormal. Letting $c: \mathrm{SU}(2) \rightarrow \mathrm{SU}(2)^{2}$ be the result of fixing $t$ in $\beta$, that is $c(A)=\beta(A, t)$, we could interpret this formula for fixed $t$ as

$$
\left\|d c_{\mathrm{Id}}\right\|\left(\beta_{(\mathrm{Id}, t)}\right)^{*} v^{X}\left(\frac{d}{d t}\right),
$$

where $v^{X}$ is the Riemannian volume form from the push-froward metric on the character variety of $\pi_{1}\left(S^{3}-N(K)\right)$ at the point $\left[\rho_{t}\right]$. Notice that $d c_{\text {Id }}$ is the first boundary operator in the complex used to define Dubois torsion.

The determinant of matrix (15) is $\pm\|d \bar{r}\|$. Finally, the last factor in the formula for torsion contributes $\|\vec{v}\|$.

Putting it all together this means that the Reidemeister torsion can be thought of as a one-form $d \tau$ on the character variety of the knot complement, given by

$$
d \tau=\frac{\|d c\|\|\vec{v}\|}{\|d \bar{r}\|} v^{X}
$$

where $d c$ is the derivative of the map given by conjugation, $d \bar{r}$ is the derivative of the relator map, and $v^{X}$ is the push-forward of the Riemannian volume from the representation variety to the character variety.

However, a more useful formulation for us takes place in the representation variety, where we see

$$
d \tau(h)=\frac{\nu^{R}\left(D \beta_{(\mathrm{Id}, t)}\right)\|\vec{v}\|}{\|d \bar{r}\|}
$$

where

$$
\beta: \mathrm{SU}(2) \times(-\varepsilon, \varepsilon) \longrightarrow R^{i}\left(S^{3}-N(K)\right)
$$

is of the form

$$
\beta(A, t)=A \rho_{t} A^{-1}
$$

and

$$
\rho_{t}:(0, \pi) \longrightarrow R^{i}\left(S^{3}-N(K)\right)
$$

is a slice of the projection mapping

$$
R^{i}\left(S^{3}-N(K)\right) \longrightarrow X\left(S^{3}-N(K)\right)
$$

over the path $\left[\rho_{t}\right]:(0, \pi) \rightarrow X\left(S^{3}-N(K)\right)$ whose derivative is the tangent vector $h$. Finally, $v^{R}$ is the Riemannian volume form on $R^{i}\left(S^{3}-N(K)\right)$ coming from its inclusion in $\mathrm{SU}(2)^{k}$. 
3.4. Construction of the seminorm. A seminorm on a vector space $V$ is a map

$$
\|\|_{s}: V \longrightarrow \mathbb{R}_{\geq 0}
$$

such that for all $\vec{u}, \vec{w} \in V$,

$$
\|\vec{u}+\vec{w}\|_{s} \leq\|\vec{u}\|_{s}+\|\vec{w}\|_{s}
$$

and, for any $\lambda \in \mathbb{R}$,

$$
\|\lambda \vec{u}\|_{s}=\left|\lambda\|\mid \vec{u}\|_{s} .\right.
$$

If $V$ is in addition a commutative algebra, we define the radical of \|\|$_{s}$ to be the set of all $\vec{u} \in V$ such that for all $\vec{w} \in V$,

$$
\|\vec{u} \vec{w}\|_{s}=0 .
$$

By design the radical of a seminorm is an ideal. Any norm is a seminorm and its radical is the zero ideal.

At a regular representation, the sequence

$$
0 \stackrel{\rho}{\longrightarrow} R\left(S^{3}-N(K)\right) \stackrel{i}{\longrightarrow} \bigoplus_{k} \operatorname{su}(2) \stackrel{\delta^{2}}{\longrightarrow} \bigoplus_{k-1} \operatorname{su}(2) \stackrel{\delta^{3}}{\longrightarrow} \mathbb{R} \longrightarrow 0
$$

is exact. This is almost the same sequence as (10). The sequences differ in the first term, and the map $i$ is an inclusion. We use different notation in the second and third terms. For instance, $\sum_{i} e^{i} \otimes w_{i} \in C^{1}(W, \operatorname{Ad} \rho)$ corresponds to the tuple $\left(w_{1}, \ldots w_{k}\right) \in \bigoplus_{k} \operatorname{su}(2)$. Using direct sums of the standard orientations on $\mathbb{R}$ and on $\operatorname{su}(2)=\mathbb{R}^{3}$, we have orientations for $\bigoplus_{k} \operatorname{su}(2)$ and $\bigoplus_{k-1} \operatorname{su}(2)$. This allows us to force an orientation on $\mathrm{T}_{\rho} R\left(S^{3}-N(K)\right)$. As $\mathrm{T}_{\rho} R\left(S^{3}-N(K)\right)$ inherits an inner product as a subspace of $\bigoplus_{k} \operatorname{su}(2)=\mathbb{R}^{3 k}$ there is a Riemannian volume $v^{R}$ on $\mathrm{T}_{\rho} R\left(S^{3}-N(K)\right)$.

The torsion of (18) defines a 4-form on $\mathrm{T}_{\rho} R\left(S^{3}-N(K)\right)$. Using the action of $\mathrm{SU}(2)$ on $R\left(S^{3}-N(K)\right)$ by conjugation we can push the 4-form down to a 1-form on $\mathrm{T}_{[\rho]} X\left(S^{3}-N(K)\right)$. The push-forward is Dubois torsion.

In our example, we could parametrize the regular representations of the fundamental group of the complement of the trefoil by taking a path of representations and conjugating them. You can do this at any regular representation of a knot group. Here is how. Parametrize $X\left(S^{3}-N(K)\right)$ near $[\rho]$ by $\left[\rho_{t}\right]:(-\varepsilon, \varepsilon) \rightarrow X\left(S^{3}-N(K)\right)$. Using a slice of the quotient map $R\left(S^{3}-N(K)\right) \rightarrow X\left(S^{3}-N(K)\right)$ lift $\left[\rho_{t}\right]$ to $\rho_{t}:(-\varepsilon, \varepsilon) \rightarrow R\left(S^{3}-N(K)\right)$. Combining with conjugation we get a local parametrization $\beta: \mathrm{SU}(2) \times(-\varepsilon, \varepsilon) \rightarrow R\left(S^{3}-N(K)\right)$,

$$
\beta(A, t)=A \rho_{t} A^{-1} .
$$


Computing $v^{R}$ in these coordinates we get that it is $\left[\overline{\mathbf{b}_{\mathbf{0}}}, \rho_{t}^{\prime} \rho_{t}^{-1}, \mathbf{b}_{\mathbf{1}} / \mathbf{c}_{\mathbf{1}}\right]$ up to sign, as long as we chose our $\mathbf{b}_{0}$ and $\mathbf{b}_{1}$ to be orthonormal. Furthermore, if we choose $\mathbf{b}_{2}$ to be orthonormal as well then $\left[\overline{\mathbf{b}_{1}}, \overline{\mathbf{b}_{2}} / \mathbf{c}_{2}\right]$ is $\|d \bar{r}\|$ up to sign, where $\bar{r}$ was defined in (6). Thus if we orient $R^{i}\left(S^{3}-N(K)\right)$ as above then the absolute value of Dubois torsion at a representation $\rho$ is

$$
\tau_{\rho}(h)=\frac{v^{R}\left(D \beta_{(\mathrm{Id}, t)}\right)\|\vec{v}\|}{\|d \bar{r}\|} .
$$

The manifold $R^{i}\left(S^{3}-N(K)\right)$ is oriented, and the local parametrizations we have chosen preserve orientation. Hence we can integrate torsion in local coordinates. By standard arguments these integrals can be woven together to yield a global value so long as the value of the integral is finite. Hence, for any function

$$
f: R^{i}\left(S^{3}-N(K)\right) \longrightarrow \mathbb{R}
$$

its integral against Dubois torsion is given by

$$
\int_{R^{i}\left(S^{3}-N(K)\right)} f d \tau=\int_{R^{i}\left(S^{3}-N(K)\right)} f \frac{\|\vec{v}\|}{\|d \bar{r}\|} v^{R},
$$

provided that it converges.

Definition 3.1. Let $f \in C\left[X\left(T^{2}\right)\right]$, using restriction we can view $f$ as a function on $R\left(S^{3}-N(K)\right)$. Define

$$
\|f\|_{s}=\left|\int_{R^{i}\left(S^{3}-N(K)\right)} f \frac{\|\vec{v}\|}{\|d \vec{r}\|} v^{R}\right|
$$

if the integral is defined.

Define the total Dubois torsion of the knot $K$ to be

$$
\tau(K)=\int_{R^{i}\left(S^{3}-N(K)\right)} d \tau .
$$

Theorem 3.2. For all knots $K$ such that the regular representations $R^{i}\left(S^{3}-N(K)\right)$ form an open dense subset of $R\left(S^{3}-N(K)\right)$ and the total Dubois torsion of $R^{i}\left(S^{3}-\right.$ $N(K))$ is finite, the seminorm \|\|$_{s}$ is an invariant of the knot complement. The radical of this invariant is the A-ideal.

Proof. This follows from the fact that when it is defined, Reidemeister torsion is never 0 , so the support of the measure defined by the Dubois torsion is the closure of the regular irreducible representations. 
In the case of torus knots, the total torsion is exactly computable. For instance the total Dubois torsion of the trefoil knot is $\frac{4 \pi}{3}$. It is a little more work, but completely elementary to compute the integral of any peripheral character against Dubois torsion. This can be done by calculating the integrals of the powers of the real part of the image of the meridian, and then proving that these are a basis for the values of the integral against Dubois torsion of the peripheral skeins.

\section{Dubois torsion and the square root of Reidemeister torsion of the double}

In this section we prove that the Dubois torsion of a knot complement is a geometrically motivated evaluation of the square root of the Reidemeister torsion of its double. When the character variety of a knot is sufficiently regular, the total Dubois torsion is proportional to the conjectured formula for the leading asymptotics of the Witten-Reshetikhin-Turaev invariant of the double of the knot complement, see the discussion in Section 6.

Let $M$ be a closed, oriented 3-manifold. Suppose that $\rho: \pi_{1}(M) \rightarrow \mathrm{SU}(2)$ is irreducible, and $h$ is a basis for $H^{1}(M ; \operatorname{Ad} \rho)$. Choose $h^{*}$ to be a basis for $H^{2}(M ; \operatorname{Ad} \rho)$ that is dual to $h$ with respect to the cup pairing

$$
\cup: H^{1}(M ; \operatorname{Ad} \rho) \otimes H^{2}(M ; \operatorname{Ad} \rho) \longrightarrow H^{3}(M ; \mathbb{R}) .
$$

Since $\rho$ is irreducible,

$$
H^{0}(M ; \operatorname{Ad} \rho) \simeq H^{3}(M ; \operatorname{Ad} \rho)=\{\overrightarrow{0}\} .
$$

Thus

$$
H^{*}(M ; \operatorname{Ad} \rho) \simeq H^{1}(M ; \operatorname{Ad} \rho) \oplus H^{2}(M ; \operatorname{Ad} \rho) .
$$

The square root of the Reidemeister torsion of $M$ evaluated on $\left\{h, h^{*}\right\}$, denoted by $\sqrt{\tau}(M ; h)$, is the square root of the Reidemeister torsion of the cochain complex $C^{*}(M ; \mathrm{Ad} \rho)$ with respect to the choice of basis $\left\{h, h^{*}\right\}$. As recognized by Rozansky [43], $\sqrt{\tau}(M ; h)$ is a naturally defined volume on the part of the character variety of $M$ coming from regular irreducible representations.

There is an approach to Reidemeister torsion due to Milnor [37] based on volumed vector spaces. A volumed vector space is a vector space $V$ along with a choice of a nonzero element of the top dimensional exterior power of the vector space, called the volume. The top dimensional exterior power of the direct sum of two vector spaces is canonically isomorphic to the tensor product of the top dimensional exterior powers of the two vector spaces. If $\left\{v_{1}, \ldots, v_{k}\right\}$ is the preferred bases for $V$ then $v_{1} \wedge v_{2} \wedge \cdots \wedge v_{k}$ is the preferred volume. 
Suppose that $A, B$ and $C$ are chain complexes defined over a field and there is a short exact sequence

$$
0 \longrightarrow A \stackrel{\alpha}{\longrightarrow} B \stackrel{\beta}{\longrightarrow} C \longrightarrow 0 .
$$

Since the chain groups are vector spaces, for each $i$ the sequence splits, so that $B_{i} \cong A_{i} \oplus C_{i}$. We say the sequence is volume exact if for each $i$ the volume on $B_{i}$ is the tensor product of the volumes on $A_{i}$ and $C_{i}$.

From the long exact sequence in cohomology derived from the short exact sequence (23), we can split the cohomology groups of $A, B$ and $C$. Let $E=\Delta(J)$, where $\Delta$ is the connecting homomorphism and $J$ is a complement to its kernel. Let $F$ be a complement to $E$, and $G=\alpha(F)$. Finally let $H$ be a complement of $G$ and $I=\beta(H)$. With these choices $H^{*}(A)=E \oplus F, H^{*}(B)=G \oplus H$ and $H^{*}(C)=I \oplus J$.

Choose bases $\mathbf{f}$ for $F, \mathbf{h}$ for $H$ and $\mathbf{j}$ for $J$. Let $\overline{\mathbf{f}}, \overline{\mathbf{h}}$, and $\overline{\mathbf{j}}$, denote their images under $\alpha, \beta$ and $\Delta$ on the chain level. The concatenations $\{\overline{\mathbf{j}}, \mathbf{f}\},\{\overline{\mathbf{f}}, \mathbf{h}\}$ and $\{\overline{\mathbf{h}}, \mathbf{j}\}$ form bases for the cohomology groups of the complexes $A, B$ and $C$ respectively. Let $\tau_{A}(\overline{\mathbf{j}}, \mathbf{f}), \tau_{B}(\overline{\mathbf{f}}, \mathbf{h}), \tau_{C}(\overline{\mathbf{h}}, \mathbf{j})$ denote the Reidemeister torsion of each complex with respect to the indicated choice of bases. The following proposition is a consequence of Theorem 3.2 in [37], or Corollary 1.2 in [17].

Proposition 4.1. $\tau_{B}(\overline{\mathbf{f}}, \mathbf{h})=\tau_{A}(\overline{\mathbf{j}}, \mathbf{f}) \tau_{C}(\overline{\mathbf{h}}, \mathbf{j})$.

In order to analyze the cohomology of the double of a knot complement we will use the Mayer-Vietoris sequence coming from the fact that the double is the union of two copies of the knot complement. Suppose that $M=A \cup B$ is excisive with inclusion map $i: A \cap B \rightarrow M$, and suppose that $\rho: \pi_{1}(M) \rightarrow \mathrm{SU}(2)$. There are two Mayer-Vietoris sequences, one for cohomology with coefficients $\operatorname{Ad} \rho$,

$$
\begin{aligned}
H^{i-1} & (A \cap B ; \operatorname{Ad} \rho) \stackrel{\Delta}{\longrightarrow} H^{i}(M ; \operatorname{Ad} \rho) \longrightarrow H^{i}(A ; \operatorname{Ad} \rho) \oplus H^{i}(B ; \operatorname{Ad} \rho) \\
& \longrightarrow H^{i}(A \cap B ; \operatorname{Ad} \rho),
\end{aligned}
$$

and the other for cohomology with untwisted coefficients $\mathbb{R}$,

$$
H^{i-1}(A \cap B ; \mathbb{R}) \stackrel{\Delta^{\prime}}{\longrightarrow} H^{i}(M ; \mathbb{R}) \longrightarrow H^{i}(A ; \mathbb{R}) \oplus H^{i}(B ; \mathbb{R}) \longrightarrow H^{i}(A \cap B ; \mathbb{R}) .
$$

The dot product $\operatorname{su}(2) \otimes \operatorname{su}(2) \rightarrow \mathbb{R}$ allows us to define a cup pairing,

$$
\cup: H^{i}(M ; \operatorname{Ad} \rho) \otimes H^{j}(M ; \operatorname{Ad} \rho) \longrightarrow H^{i+j}(M ; \mathbb{R}) .
$$

For any $\alpha \in H^{i}(A \cap B ; \operatorname{Ad} \rho)$ and $\beta \in H^{j}(M ; \operatorname{Ad} \rho)$ we have

$$
\Delta(\alpha) \cup \beta=\Delta^{\prime}\left(\alpha \cup i^{*} \beta\right) .
$$

This formula is a consequence of the naturality of the connecting homomorphism along with the fact that the dot product is symmetric and Ad-invariant. 
Suppose now that $S^{3}-N(K)$ is a knot complement with boundary $T^{2}$. Denote by $M$ the double of $S^{3}$, so that $M=X_{1} \cup X_{2}$, where $X_{1}$ and $X_{2}$ are two copies of $S^{3}-N(K)$, with the orientation of $X_{2}$ the opposite from the orientation on $S^{3}-N(K)$, and $X_{1}$ and $X_{2}$ identified along their boundaries via the identity map. There is a regular CW-decomposition of $S^{3}-N(K)$ with one vertex $v$ on the boundary, which can be doubled to get a CW-decomposition of $M$.

Let $\rho$ be a regular $\mathrm{SU}(2)$ representation of $\pi_{1}\left(S^{3}-N(K)\right)$. Let $\mu$ denote the meridian of $S^{3}-N(K)$ and let $\rho(\mu)=\cos \varphi+\sin \varphi \vec{P}$. Let

$$
g=\exp (\theta \vec{P})
$$

where $0 \leq \theta \leq \pi$. Let

$$
\rho_{g}: \pi_{1}(M) \rightarrow \mathrm{SU}(2)
$$

be the representation that restricts to $\rho$ on $\pi_{1}\left(X_{2}\right)$ and to $g \rho g^{-1}$ on $\pi_{1}\left(X_{1}\right)$. The construction of a path of representations depending on the variable $\theta$ like this is called bending. The tangent vector to such a path can be thought of as a tangent vector to a deformation. Note that $C^{*}\left(S^{3}-N(K)\right.$; $\left.\mathrm{Ad}\left(g \rho g^{-1}\right)\right)$ is canonically isomorphic to $C^{*}\left(S^{3}-N(K)\right.$; Ad $\left.\rho\right)$, under the isomorphism $c^{*} \otimes \vec{v} \mapsto c^{*} \otimes g . \vec{v}$. By dint of this isomorphism we identify $C^{*}\left(X_{1} ; \operatorname{Ad}\left(g \rho g^{-1}\right)\right)$ and $C^{*}\left(X_{2} ; \operatorname{Ad} \rho\right)$ with $C^{*}\left(S^{3}-\right.$ $N(K) ; \operatorname{Ad} \rho)$.

In order to relate the torsion of the knot complement to the square root of the torsion of the double we need to choose the bases for the cohomology groups with coefficients in Ad $\rho$, for all the spaces in the Mayer-Vietoris sequence for $M=X_{1} \cup X_{2}$.

Since $\rho$ restricted to the boundary is not central, we have $H^{*}\left(T^{2} ; \operatorname{Ad} \rho\right)=$ $H^{*}\left(T^{2} ; \mathbb{R}\right) \otimes \mathbb{R} \vec{P}$. A volume basis for $H^{*}\left(T^{2} ; \operatorname{Ad} \rho\right)$ can be chosen as follows. Denote by $v^{*}$ a cochain in $C^{0}\left(T^{2} ; \mathbb{R}\right)$ dual to the vertex $v$, and let $t \in C^{2}\left(T^{2} ; \mathbb{R}\right)$ be a cochain with $t\left[T^{2}\right]=1$. Finally, let $\xi$ and $\eta$ be cocycles in $C^{1}\left(T^{2} ; \mathbb{R}\right)$ such that $\xi \cup \eta=t$. A volume basis consists of $v^{*} \otimes \vec{P}, \xi \otimes \vec{P}, \eta \otimes \vec{P}$ and $t \otimes \vec{P}$.

Since $\rho$ is regular, $H^{1}\left(S^{3}-N(K)\right.$; $\left.\operatorname{Ad} \rho\right)$ is one-dimensional and is spanned by the tangent vector $h$ to a path of representations. The restriction map

$$
H^{2}\left(S^{3}-N(K) ; \operatorname{Ad} \rho\right) \longrightarrow H^{2}\left(T^{2} ; \operatorname{Ad} \rho\right)
$$

is an isomorphism. Finally $H^{0}\left(S^{3}-N(K)\right.$; $\left.\operatorname{Ad} \rho\right)=0$ as $\rho$ is irreducible.

From this we conclude that $H^{1}\left(M ; \mathrm{Ad} \rho_{g}\right)$ is two-dimensional. One dimension comes from the image of $H^{0}\left(T^{2} ; \mathrm{Ad} \rho\right)$ under the connecting homomorphism $\Delta$. The second is accounted for by the fact that any complement of the image of $\Delta$ is mapped injectively into the diagonal of the direct sum

$$
\begin{aligned}
& H^{1}\left(X_{1} ; \operatorname{Ad}\left(g \rho g^{-1}\right)\right) \oplus H^{1}\left(X_{2} ; \operatorname{Ad} \rho\right) \\
& \quad=H^{1}\left(S^{3}-N(K) ; \operatorname{Ad} \rho\right) \oplus H^{1}\left(S^{3}-N(K) ; \operatorname{Ad} \rho\right) .
\end{aligned}
$$


The second cohomology of $M$ is also two-dimensional where analogously one dimension is accounted for by the image of the connecting homomorphism and any complement of that image gets mapped injectively into the diagonal of

$$
\begin{aligned}
& H^{2}\left(X_{1} ; \operatorname{Ad}\left(g \rho g^{-1}\right)\right) \oplus H^{2}\left(X_{2} ; \operatorname{Ad} \rho\right) \\
& \quad=H^{2}\left(S^{3}-N(K) ; \operatorname{Ad} \rho\right) \oplus H^{2}\left(S^{3}-N(K) ; \operatorname{Ad} \rho\right) .
\end{aligned}
$$

There is a nondegenerate pairing coming from the cup product on $M$ and the dot product on the coefficients,

$$
\cup: H^{1}\left(M ; \operatorname{Ad} \rho_{g}\right) \otimes H^{2}\left(M ; \operatorname{Ad} \rho_{g}\right) \longrightarrow H^{3}(M ; \mathbb{R}) .
$$

It should be noted that if $m \in H^{3}(M ; \mathbb{R})$ is a cohomology class with $m([M])=1$ then $\Delta^{\prime}(t)=m$ where $\Delta^{\prime}$ is the connecting homomorphism from the Mayer-Vietoris sequence for $M=X_{1} \cup X_{2}$ with real coefficients.

If $t^{\prime} \in H^{2}\left(S^{3}-N(K) ; \operatorname{Ad} \rho\right)$ is chosen so that restriction of $t^{\prime}$ to $H^{2}\left(T^{2} ; \operatorname{Ad} \rho\right)$ is $t$ and $h$ is a basis for $H^{1}\left(S^{3}-N(K)\right.$; Ad $\left.\rho\right)$ then the Dubois torsion $\tau\left(S^{3}-N(K) ; h\right)$ is the Reidemeister torsion of $C^{*}\left(S^{3}-N(K)\right.$; $\left.\operatorname{Ad} \rho\right)$ with respect to the bases $h$ for $H^{1}\left(S^{3}-N(K) ; \operatorname{Ad} \rho\right)$ and $t^{\prime}$ for $H^{2}\left(S^{3}-N(K) ; \operatorname{Ad} \rho\right)$.

Theorem 4.2. Let $M$ be the double of $S^{3}-N(K)$ and let $\rho_{g}: \pi_{1}(M) \rightarrow \mathrm{SU}(2)$ be obtained from bending $\rho: \pi_{1}\left(S^{3}-N(K)\right) \rightarrow \mathrm{SU}(2)$ as above. Assume that $\rho$ and $\rho_{g}$ are regular. Choose $h$ as the basis for $H^{1}\left(S^{3}-N(K)\right.$; Ad $\left.\rho\right)$ and choose $\tilde{h} \in$ $H^{1}(M ; \operatorname{Ad} \rho)$ that restricts to $h$. If we use the basis $\left\{\Delta\left(v^{*} \otimes \vec{P}\right), \tilde{h}\right\}$ for $H^{1}(M ; \operatorname{Ad} \rho)$, then

$$
\sqrt{\tau}\left(M ;\left\{\Delta\left(v^{*} \otimes \vec{P}\right), \tilde{h}\right\}\right)=\tau\left(S^{3}-N(K) ; h\right) .
$$

Proof. The proof is an application of Proposition 4.1 followed by some interpretation. We apply the proposition to the short exact sequence inducing the Mayer-Vietoris sequence

$$
\begin{aligned}
0 \longrightarrow & C^{*}\left(M ; \operatorname{Ad} \rho_{g}\right) \stackrel{\left(j_{1}^{*}, j_{2}^{*}\right)}{\longrightarrow} C^{*}\left(X_{1} ; \operatorname{Ad}\left(g \rho g^{-1}\right)\right) \oplus C^{*}\left(X_{2} ; \operatorname{Ad} \rho\right) \\
& \stackrel{\left(i_{1}^{*},-i_{2}^{*}\right)}{\longrightarrow} C^{*}\left(T^{2} ; \operatorname{Ad} \rho\right) \longrightarrow 0
\end{aligned}
$$

where $j_{1}^{*}, j_{2}^{*}$ are induced by restrictions from $M$ to the two knot complements, and $i_{1}^{*}, i_{2}^{*}$ are induced by restrictions to the boundary torus. These are the chain complexes associated to a regular CW-decomposition of $S^{3}-N(K)$ with a single vertex $v$ on the boundary, which we double to get a CW-decomposition of $M$. We use the inner product on $C^{*}(M ; \operatorname{Ad} \rho)$, coming from the $\mathrm{CW}$-structure and the dot product on $\operatorname{su}(2)=\mathbb{R}^{3}$. Sequence (26) is volume exact. 
Denote by $\tilde{h}$ a cocycle in $C^{1}(M ; \operatorname{Ad} \rho)$ that restricts to the basis $h$ chosen for $H^{1}\left(S^{3}-N(K)\right.$; Ad $\left.\rho\right)$. We use the basis $\left\{\Delta\left(v^{*} \otimes \vec{P}\right), \tilde{h}\right\}$ for $H^{1}\left(M ; \operatorname{Ad} \rho_{g}\right)$. The pair $\{(h, h),(h / 2,-h / 2)\}$ forms a basis for

$$
\begin{aligned}
& H^{1}\left(X_{1} ; \operatorname{Ad}\left(g \rho g^{-1}\right)\right) \oplus H^{1}\left(X_{2} ; \operatorname{Ad} \rho\right) \\
& \quad=H^{1}\left(S^{3}-N(K) ; \operatorname{Ad} \rho\right) \oplus H^{1}\left(S^{3}-N(K) ; \operatorname{Ad} \rho\right) .
\end{aligned}
$$

Notice that the vector $(h, h)$ is the image of $\tilde{h}$ under the map in the Mayer-Vietoris sequence. Denote the image of $(h / 2,-h / 2)$ in $H^{1}\left(T^{2}\right.$; Ad $\left.\rho\right)$ under $\left(i_{1}^{*}, i_{2}^{*}\right)$ by $t_{1}$. Note that $t_{1}$ is a tangent vector to the image under restriction of the curve of regular characters $X^{i}\left(S^{3}-N(K)\right)$ in the pillowcase.

Let $n$ be a normal vector to $X^{i}\left(S^{3}-N(K)\right)$ at $[\rho]$, so that $\left\{t_{1}, n\right\}$ is a volume basis for $H^{1}\left(T^{2} ; \operatorname{Ad} \rho\right)$. Let $h^{\prime} \in C^{2}\left(S^{3}-N(K)\right.$; Ad $\left.\rho\right)$ be a cocycle that restricts to $t \otimes \vec{P}$ in $H^{2}\left(T^{2}\right.$; Ad $\left.\rho\right)$. We use $\left\{\left(h^{\prime}, h^{\prime}\right),\left(h^{\prime} / 2,-h^{\prime} / 2\right)\right\}$ as a basis for

$$
\begin{aligned}
& H^{2}\left(X_{1} ; \operatorname{Ad}\left(g \rho g^{-1}\right)\right) \oplus H^{2}\left(X_{2} ; \operatorname{Ad} \rho\right) \\
& \quad=H^{2}\left(S^{3}-N(K) ; \operatorname{Ad} \rho\right) \oplus H^{2}\left(S^{3}-N(K) ; \operatorname{Ad} \rho\right) .
\end{aligned}
$$

Let $h_{2} \in C^{2}\left(M ; \operatorname{Ad} \rho_{g}\right)$ be a cocycle that pulls back to $\left(h^{\prime}, h^{\prime}\right)$. Finally use $\left\{\Delta(n), h_{2}\right\}$ as the basis for $H^{2}\left(M ; \operatorname{Ad} \rho_{g}\right)$.

We have chosen our bases as in the hypothesis of Proposition 4.1. Thus

$$
\begin{aligned}
& \tau\left(M ;\left\{\Delta\left(v^{*} \otimes \vec{P}\right), \tilde{h}, \Delta(n), h_{2}\right\}\right) \\
& =\tau\left(T^{2},\left\{v^{*} \otimes \vec{P}, t_{1}, n, t\right\}\right) . \\
& \tau\left(C^{*}\left(X_{1} ; \operatorname{Ad} \rho\right) \oplus C^{*}\left(X_{2} ; \operatorname{Ad} \rho\right)\right. \text {; } \\
& \left.\left\{(h, h),(h / 2,-h / 2),\left(h^{\prime}, h^{\prime}\right),\left(h^{\prime} / 2,-h / 2\right)\right\}\right) \text {. }
\end{aligned}
$$

We chose the basis for $T^{2}$ to be a volume basis, so

$$
\tau\left(T^{2},\left\{v^{*} \otimes \vec{P}, t_{1}, n, t\right\}\right)=1
$$

We need to show that the basis $\left\{\Delta\left(v^{*} \otimes \vec{P}\right), \tilde{h}, \Delta(n), h_{2}\right\}$ for $H^{*}(M ; \operatorname{Ad} \rho)$ can be used to compute

$$
\left(\sqrt{\tau}\left(M ;\left\{\Delta\left(v^{*} \otimes \vec{P}\right), \tilde{h}\right\}\right)\right)^{2} .
$$


This entails showing that the volume form on $H^{2}(M ; \operatorname{Ad} \rho)$ induced by $\left\{\Delta(n), h_{2}\right\}$ coincides with the volume form induced by the basis dual to $\left\{\Delta\left(v^{*} \otimes \vec{P}\right), \tilde{h}\right\}$. This is done by showing that the determinant of the matrix of cup pairings is equal to 1 . To see this we need to compute the four cup pairings

$$
\begin{gathered}
\Delta^{0}\left(v^{*} \otimes \vec{P}\right) \cup \Delta^{1}(n), \\
\Delta^{0}\left(v^{*} \otimes \vec{P}\right) \cup h_{2}, \\
\tilde{h} \cup \Delta^{1}(n),
\end{gathered}
$$

and

$$
\tilde{h} \cup h_{2} .
$$

We do this by repeated applications of (24). The first cup product is

$$
\Delta^{0}\left(v^{*} \otimes \vec{P}\right) \cup \Delta^{1}(n)=\Delta^{1}\left(v^{*} \otimes \vec{P} \cup i^{*} \Delta^{1}(n)\right)=0,
$$

as $i^{*} \Delta^{1}(n)=0$. The second cup product is

$$
\Delta^{0}\left(v^{*} \otimes \vec{P}\right) \cup h_{2}=\Delta^{2}\left(v^{*} \otimes \vec{P} \cup i^{*} h_{2}\right)=1,
$$

as $i^{*} h_{2}=t \otimes \vec{P}$. The third cup product is

$$
\tilde{h} \cup \Delta^{1}(n)=\Delta^{1}(n) \cup \tilde{h}=\Delta^{2}\left(n \cup i^{*} \tilde{h}\right)=-1,
$$

since $i^{*} \tilde{h}=t_{1}$. We do not care what the last pairing is because

$$
\operatorname{det}\left(\begin{array}{cc}
0 & 1 \\
-1 & *
\end{array}\right)=1
$$

for any $*$. Thus it is possible to make a volume preserving change of basis from $\left\{\Delta\left(v^{*} \otimes \vec{P}\right), \tilde{h}, \Delta(n), h_{2}\right\}$ to get the basis formed by $\left\{\Delta\left(v^{*} \otimes \vec{P}\right), \tilde{h}\right\}$ and its dual. We showed that

$$
\tau\left(M ;\left\{\Delta\left(v^{*} \otimes \vec{P}\right), \tilde{h}, \Delta(n), h_{2}\right\}\right)=\left(\sqrt{\tau}\left(M ;\left\{\Delta\left(v^{*} \otimes \vec{P}\right), \tilde{h}\right\}\right)\right)^{2}
$$

Finally, we interpret

$$
\tau\left(C^{*}\left(X_{1} ; \operatorname{Ad} \rho\right) \oplus C^{*}\left(X_{2} ; \operatorname{Ad} \rho\right) ;\left\{(h, h),(h / 2,-h / 2),\left(h^{\prime}, h^{\prime}\right),\left(h^{\prime} / 2,-h^{\prime} / 2\right)\right\}\right)
$$

as the square of the Dubois torsion of the knot complement. Change bases by elementary row operations that do not change volume to get

$$
\begin{gathered}
\tau\left(C^{*}\left(X_{1} ; \operatorname{Ad} \rho\right) \oplus C^{*}\left(X_{2} ; \operatorname{Ad} \rho\right) ;\left\{(h, h),(h / 2,-h / 2),\left(h^{\prime}, h^{\prime}\right),\left(h^{\prime} / 2,-h^{\prime} / 2\right)\right\}\right) \\
=\tau\left(C^{*}\left(X_{1} ; \operatorname{Ad} \rho\right) \oplus C^{*}\left(X_{2} ; \operatorname{Ad} \rho\right) ;\left\{(h, 0),(0,-h),\left(h^{\prime}, 0\right),\left(0,-h^{\prime}\right)\right\}\right) .
\end{gathered}
$$


Since the basis $\left\{(h, 0),(0,-h),\left(h^{\prime}, 0\right),\left(0,-h^{\prime}\right)\right\}$ is a direct sum of bases for the spaces $H_{*}\left(X_{1} ; \operatorname{Ad} \rho\right)$ and $H^{*}\left(X_{2} ; \operatorname{Ad} \rho\right)$, the torsion now splits as the product of torsions, which both compute the Dubois torsion of $S^{3}-N(K)$ at $\rho$,

$$
\begin{aligned}
& \tau\left(C^{*}\left(X_{1} ; \operatorname{Ad} \rho\right) \oplus C^{*}\left(X_{2} ; \operatorname{Ad} \rho\right) ;\left\{(h, 0),(0,-h),\left(h^{\prime}, 0\right),\left(0,-h^{\prime}\right)\right\}\right) \\
& \quad=\tau\left(X_{1} ; h\right) \tau\left(X_{2} ; h^{\prime}\right) \\
& \quad=\left(\tau\left(S^{3}-N(K) ; h\right)\right)^{2} .
\end{aligned}
$$

Substituting (28), (29), and (30) into (27) we obtain the desired result.

To understand Theorem 4.2 geometrically, consider a path of representations of $\pi_{1}\left(S^{3}-N(K)\right)$ that covers an arc of regular characters. Denote this path by $Y\left(S^{3}-\right.$ $N(K))$. Bending gives rise to a circle bundle over $Y\left(S^{3}-N(K)\right)$ in the representation variety of the fundamental group of the double of the knot complement. Call this bundle $Y(M)$. Denote the projection that restricts a representation of the double to the second copy of the knot complement by

$$
\sigma: Y(M) \longrightarrow Y\left(S^{3}-N(K)\right) .
$$

The derivative of $\sigma$ can be understood as a map

$$
d \sigma: H^{1}(M ; \operatorname{Ad} \rho) \longrightarrow H^{1}\left(S^{3}-N(K) ; \operatorname{Ad} \rho\right) .
$$

The kernel of $d \sigma$ is the span of $\Delta^{0}\left(v^{*} \times \vec{P}\right)$. The partial derivative of the parametrization of $Y(M)$ by bending, with respect to the variable $\theta$ defined in (25), is equal to $\Delta^{0}\left(v^{*} \times \vec{P}\right)$.

Proposition 4.3. The measure on $Y\left(S^{3}-N(K)\right)$ coming from Dubois torsion is $\frac{1}{\pi}$ times the pushforward measure under $\sigma$ of the measure on $Y(M)$ coming from the square root of Reidemeister torsion.

Proof. The volume of the fiber is $\pi\left\|\Delta^{0}\left(v^{*} \times \vec{P}\right)\right\|$ which is $\pi\|\vec{v}\|$, where $\vec{v}$ is the vector defined by (12). If $S \subset Y\left(S^{3}-N(K)\right)$ is measurable, then the measure of $\sigma^{-1}(S)$ is $\pi$-times the measure of $S$.

Remark 4.4. The factor $\|\vec{v}\|$ that appears in (13) and in (17) for Dubois torsion can be attributed to partial integration over the fiber of the map $\sigma$ from (31).

Assume now that $K \subset S^{3}$ is a knot such that the regular representations are dense in the nonabelian representations, and the map $X\left(S^{3}-N(K)\right) \rightarrow X\left(T^{2}\right)$ is an embedding away from finitely many points. The character variety of $M$ has a component coming from bent representations lying over each component coming from regular representations of $\pi_{1}\left(S^{3}-N(K)\right)$. 
Proposition 4.5. Let $Y^{i}(M)$ be the part of the representation variety of $M$ that comes from bending regular representations of $\pi_{1}\left(S^{3}-N(K)\right)$. Let

$$
f: R^{i}\left(S^{3}-N(K)\right) \longrightarrow \mathbb{R}
$$

be integrable, then

$$
\int_{Y^{i}(M)} f \sqrt{\tau}(M)=\pi \int_{R^{i}\left(S^{3}-N(K)\right)} f d \tau,
$$

where we lift $f$ to $Y^{i}(M)$ via the projection map.

\section{A global computation of the seminorm}

The aim of this section is to derive a global formula for integrating against Reidemeister torsion. We introduce a function on $\mathrm{SU}(2)^{k}$ that involves a parametrix of the heat kernel trace, and show that the limit of integrals against that function yields the seminorm defined in Section 3. The proof involves an auxiliary family of integrals to which Laplace's method can be applied. Finally, we replace the parametrix of the heat kernel with the heat kernel trace, to obtain a global formula. The local evaluation of the seminorm allows us to interpret it geometrically, while the global formula exhibits similarity to quantum invariants.

5.1. Two pointwise close Dirac delta functions. We are working with Cartesian products of $\mathrm{SU}(2)$ and intervals in the real line. We treat $\mathrm{SU}(2)$ as the unit sphere in $\mathbb{R}^{4}$. The volume form $v^{\mathrm{SU}(2)}$ from the Riemannian metric is $2 \pi^{2}$ times Haar measure. A good parametrization of SU(2) is given by three angles $(\varphi, \psi, \theta)$, where $\varphi$ and $\psi$ vary from 0 to $\pi$, and $\theta$ varies from 0 to $2 \pi$. The parametrization is

$$
X(\varphi, \psi, \theta)=(\cos \varphi, \sin \varphi \sin \psi \cos \theta, \sin \varphi \sin \psi \sin \theta, \sin \varphi \cos \psi) .
$$

The angle $\varphi$ is the angle that $X(\varphi, \psi, \theta)$ makes with $(1,0,0,0)=\mathbf{1}$. The projection of $X(\varphi, \psi, \theta)$ into the perpendicular to $(1,0,0,0)$ is a vector in $\mathbb{R}^{3}$ with length $\sin \varphi$ that makes an angle $\psi$ with the $z$-axis, and whose projection into the $x y$-plane makes an angle $\theta$ with positive $x$-axis. The volume form from the Riemannian metric inherited from $\mathbb{R}^{4}$ is

$$
\nu^{\mathrm{SU}(2)}=\sin ^{2} \varphi \sin \psi d \varphi \wedge d \psi \wedge d \theta .
$$

There are two approximate Dirac delta functions of $\mathbf{1}$ with respect to the measure $v^{\mathrm{SU}(2)}$ we would like to use. The first is

$$
\beta_{\lambda}(\varphi, \psi, \theta)=\left(\frac{\lambda}{4 \pi}\right)^{3 / 2} e^{-\lambda \varphi^{2} / 4}
$$


There is a very nice coordinate system for working with this Gaussian. Recall the exponential map, exp: $\mathbb{R}^{3} \rightarrow S^{3}$, given by

$$
\exp (\vec{w})=\cos \|\vec{w}\|+\sin \|\vec{w}\| \frac{\vec{w}}{\|\vec{w}\|} .
$$

When restricted to the open ball of radius $\pi$ centered at the origin, $B_{\pi}(\overrightarrow{0})$, the exponential map is a diffeomorphism onto $S^{3}-\{-\mathbf{1}\}$, and has an inverse

$$
\log : S^{3}-\{-\mathbf{1}\} \rightarrow B_{\pi}(\overrightarrow{0}),
$$

which is a normal coordinate chart. In these coordinates,

$$
\beta_{\lambda}(\vec{w})=\left(\frac{\lambda}{4 \pi}\right)^{3 / 2} e^{-\lambda\|\vec{w}\|^{2} / 4}
$$

Suppose that $r: M \rightarrow \mathrm{SU}(2)$ is a smooth function from a smooth manifold $M$ so that $r(p)=\mathbf{1}$, and let $U$ be a coordinate chart with coordinates $x^{i}$ at $p$, so that $w=\log (r)$ is defined in $U$. Since $\exp (w)=r$ we have that for any $\left.\frac{\partial}{\partial x^{i}}\right|_{p}$,

$$
\left.\frac{\partial}{\partial x^{i}} r\right|_{p}=\left.\frac{\partial}{\partial x^{i}} \exp (w)\right|_{p}=\exp (w(p)) \frac{\partial}{\partial x^{i}} w .
$$

Since $\exp (w(p))=r(p)=\mathbf{1}$, we have that

$$
\left.D r\right|_{p}=\left.D w\right|_{p}
$$

The second approximate Dirac delta function is the heat kernel trace $\kappa_{\lambda}$. The reason for working with $\mathrm{SU}(2)$ as the 3 -sphere in $\mathbb{R}^{4}$ is to have the eigenvalues of the Laplace operator be integers. In the $(\varphi, \psi, \theta)$ coordinates,

$$
\kappa_{\lambda}(\varphi, \psi, \theta)=\frac{1}{2 \pi^{2}} \sum_{c=0}^{\infty}(-1)^{c}(c+1) e^{-c(c+2) / \lambda} s_{c}(-2 \cos \varphi),
$$

where $s_{c}$ is the $c$-th Chebyshev polynomial defined by the recursion, $s_{0}=1, s_{1}=x$, and $s_{n}=x s_{n-1}-s_{n-2}$. In fact,

$$
s_{c}(-2 \cos \varphi)=(-1)^{c} \operatorname{tr}_{c}
$$

where $\operatorname{tr}_{c}$ is the trace in the $(c+1)$-dimensional irreducible representation of $\mathrm{SU}(2)$. These Chebyshev polynomials are the same as the ones used to define the colored Jones polynomial.

The relationship between these two approximate Dirac delta functions is that the first is the parametrix for the heat kernel trace and the second is the heat kernel trace. By design they are pointwise close. 
Lemma 5.1. For every $\varepsilon>0$ there exists $C>0$ and $U$ a neighborhood of 1 in $\mathrm{SU}(2)$ so that for $\lambda \geq C$, and $A \in U$,

$$
\left|\beta_{\lambda}(A)-\kappa_{\lambda}(A)\right|<\varepsilon .
$$

Proof. This follows directly from lemma 3.18 of [42], by substituting $\lambda$ for $1 / t$.

The fact that these two Dirac delta functions are asymptotically equivalent is sometimes called Migdal's equivalence [36]. We were first exposed to this type of local-global argument by reading [4]. Recently Bonzom and Smerlak used the same approach to understand the Reidemeister torsion of two complexes [3].

5.2. Laplace's method. Let $M$ be a Riemannian manifold with Riemannian volume form $v^{M}$. Let $\Phi: M \rightarrow \mathbb{R}$ be a nonnegative function that takes on the value 0 along the codimension $k$ submanifold $N$ with induced Riemannian volume form $v^{N}$. Let $H_{N}(\Phi)$ be the restriction of the Hessian of $\Phi$ to the normal space to $N$. Assume that $H_{N}(\Phi)$ is nondegenerate at each point in $N$, and that there is an $\varepsilon$-neighborhood of $N$ outside of which the function $\Phi$ is bounded away from 0 . It is an easy computation in local coordinates to see that if the integral on the left exists for the smooth function $f: M \rightarrow \mathbb{R}$, then

$$
\lim _{\lambda \rightarrow \infty}\left(\frac{\lambda}{\pi}\right)^{k / 2} \int_{M} f e^{-\lambda \Phi} v^{M}=\int_{N} \frac{f v^{N}}{\sqrt{\left(\operatorname{det}\left(H_{N}\right)\right)}} .
$$

Equation (35) is sometimes referred to as Laplace's method; see [51], and [2].

We will need to use Laplace's method in a slightly more general context. Given a smooth nonnegative real-valued function $\Phi$ defined on a subset of $M$, let $N=\{p \in$ $M \mid \Phi(p)=0\}$. Let $\Sigma$ be the subset of $M$ consisting of three types of points:

- points where $\Phi$ is not defined;

- points where $N$ is not a manifold;

- points where $N$ is a manifold but the Hessian of $\Phi$ restricted to the normal direction to $N$ is degenerate.

Let $U_{\varepsilon}$ be an epsilon neighborhood of $\Sigma$. If the limit

$$
C(\varepsilon)=\lim _{\lambda \rightarrow \infty}\left(\frac{\lambda}{\pi}\right)^{k / 2} \int_{U_{\varepsilon}} e^{-\lambda \Phi} v^{M}
$$

exists, and $\lim _{\varepsilon \rightarrow 0} C(\varepsilon)=0$ then we say that the singularities of $\Phi$ are tame.

Proposition 5.2. Let $M$ be compact. Suppose that the singularities $\Sigma$ of a smooth, nonnegative, real-valued function $\Phi$ defined on a subset of $M$ are tame and let $f: M \rightarrow \mathbb{R}$ be smooth; then

$$
\lim _{\lambda \rightarrow \infty}\left(\frac{\lambda}{\pi}\right)^{k / 2} \int_{M} f e^{-\lambda \Phi} v^{M}=\int_{N-\Sigma} \frac{f v^{N}}{\sqrt{\left(\operatorname{det}\left(H_{N}\right)\right)}} .
$$


5.3. The integral. We will define a function $\Phi$ whose maximum occurs along representations of the knot complement. We then use Laplace's method to compute an appropriately normalized limit of an integral of this function. The answer turns out to be the seminorm defined by the Dubois torsion. The function $\Phi$ depends on an additional parameter, which can be integrated out to recognize the parametrix of the heat kernel, yielding a local formula for integrating against the Dubois torsion. Using Migdal's equivalence we replace the parametrix of the heat kernel by the heat kernel trace, which gives us a global formula for the Dubois torsion.

Recall that given a knot $K \subset S^{3}$, we denoted the regular representations of $\pi_{1}\left(S^{3}-N(K)\right)$ into SU(2) by $R^{i}\left(S^{3}-N(K)\right)$ and the abelian representations of $\pi_{1}\left(S^{3}-N(K)\right)$ into $S \mathrm{U}(2)$ by $\mathrm{Ab}\left(S^{3}-N(K)\right)$. We work with a 2-complex embedded in the complement of the knot $K$ as a deformation retract, giving rise to $k-1$ relator maps, denoted $\left(r_{1}, \ldots, r_{k-1}\right)$, corresponding to the two-cells $\left(f_{1}, \ldots, f_{k-1}\right)$. Define the set $S \subset \mathrm{SU}(2)^{k}$ as the locus along which any of the relators takes on the value -1 . The set $S$ has measure zero as long as there is some regular representation at which the variety $R^{i}\left(S^{3}-N(K)\right)$ has dimension 4 . For a knot in $S^{3}$ this is equivalent to the statement that the set of regular representations $R^{i}\left(S^{3}-N(K)\right)$ is nonempty.

Notice that $\vec{v}$ defined by (12) can be described by word maps that are well defined for all points in $\mathrm{SU}(2)^{k}$. Let

$$
\bar{v}=\frac{1}{\left\|\left(\vec{v}_{1}, \ldots, \vec{v}_{k-1}\right)\right\|^{2}} \sum_{j} f^{j} \otimes \vec{v}_{j} .
$$

The normalized vector $\bar{v}$ is not defined on a set consisting of points where $\vec{v}=\overrightarrow{0}$. Call this set of points $T$. Thus the vector $\bar{v}$ can be extended to a function

$$
\bar{v}: \mathrm{SU}(2)^{k}-T \longrightarrow \mathrm{su}(2)^{k-1} \text {. }
$$

If the set of regular representations of the fundamental group of the knot is nonempty then $T$ has measure 0 .

Notice that $R^{i}\left(S^{3}-N(K)\right) \cap T=\emptyset$ and $\operatorname{Ab}\left(S^{3}-N(K)\right) \subset T$. This is because

$$
\vec{v}=\left(\left(\operatorname{Ad}_{\rho\left(s_{1}\right)}-\operatorname{Ad}_{\rho\left(t_{1}\right)}\right) \cdot \vec{P}, \ldots,\left(\operatorname{Ad}_{\rho\left(s_{k-1}\right)}-\operatorname{Ad}_{\rho\left(t_{k-1}\right)}\right) \cdot \vec{P}\right),
$$

where $s_{i}, t_{i}$ where defined by (11), and the vector $\vec{P}=\frac{\operatorname{Im} \rho(\mu)}{\|\operatorname{Im} \rho(\mu)\|}$, where $\mu$ is the meridian of $K$. At an abelian representations the vector $\vec{P}$ is fixed by $\operatorname{Ad}_{\rho(\gamma)}$ for all $\gamma \in \pi_{1}\left(S^{3}-N(K)\right)$. Thus the vector $\vec{v}=\overrightarrow{0}$, and its norm is zero for any abelian representation.

In our definition of the function $\Phi$ below we will use the tuple notation as opposed to the tensor used in (12). Thus

$$
\|\vec{v}\|=\left\|\left(\vec{v}_{1}, \ldots \vec{v}_{k-1}\right)\right\|,
$$

and

$$
\bar{v}=\frac{1}{\|\vec{v}\|^{2}}\left(\vec{v}_{1}, \ldots \vec{v}_{k-1}\right)=\left(\bar{v}_{1}, \ldots, \bar{v}_{k-1}\right)
$$


Fixing a relator $r_{i}$, let $\vec{w}_{i}=\log r_{i}$. We have that all $\vec{w}_{i}$ are well defined as functions from $\mathrm{SU}(2)^{k}-S$ to $\mathbb{R}^{3}$. Since each $\bar{v}_{i}$ is well defined as a function from $\mathrm{SU}(2)^{k}-T$ to $\mathbb{R}^{3}$ we can put it all together to define

$$
\Phi=\frac{\sum_{i}\left\|\vec{w}_{i}\right\|^{2}+t^{2}\left\|\bar{v}_{i}\right\|^{2}}{4}:\left(\operatorname{SU}(2)^{k}-(S \cup T)\right) \times \mathbb{R} \rightarrow \mathbb{R} .
$$

The function $\Phi$ attains its global minimum along $R^{i}\left(S^{3}-N(K)\right) \times\{0\}$, and that minimum is equal to 0 . We can apply Proposition 5.2 to the computation of the appropriately normalized limit of an integral of this function, which yields the following formula.

Theorem 5.3 (Local computation). Let $K$ be a knot in $S^{3}$ such that the regular representations $R^{i}\left(S^{3}-N(K)\right)$ form an open dense subset of $R\left(S^{3}-N(K)\right)$ and the total Dubois torsion of the knot $K$ is finite. Suppose that the map $\Phi$ defined by (40) above has tame singularities. Let $f: \mathrm{SU}(2)^{k} \rightarrow \mathbb{R}$ be any smooth invariant function. Then

$$
\int_{R^{i}\left(S^{3}-N(K)\right)} f d \tau=\lim _{\lambda \rightarrow \infty} \sqrt{\frac{4 \pi}{\lambda}} \int_{\mathrm{SU}(2)^{k}} 2^{(3 k-3) / 2} f \prod_{j} \beta_{\lambda}\left(r_{j}\right)\|\vec{v}\| v^{k} .
$$

Here $\beta_{\lambda}$ is the Dirac delta function defined in Section 5.1, the maps $r_{j}$ are the relator maps, vector $\vec{v}$ is given by (12) and (37), $v^{k}$ is the volume form from the inclusion of $\mathrm{SU}(2)^{k}$ into $\mathbb{R}^{4 k}$, and $d \tau$ is the measure from Dubois torsion.

Proof. Let $f: \mathrm{SU}(2)^{k} \rightarrow \mathbb{R}$ be a smooth function. Consider the integral,

$$
\left(\frac{\lambda}{4 \pi}\right)^{(3 k-3) / 2} \int_{\mathrm{SU}(2)^{k} \times \mathbb{R}} f e^{-\lambda \Phi} \nu^{k} d \mathscr{L},
$$

where $d \mathscr{L}$ is the Lebesgue measure on the real line.

If the singularities of $\Phi$ are tame, then we can evaluate the limit of the integral (42) as $\lambda$ goes to infinity via Laplace's method, as in Proposition 5.2. Hence, we need to compute the Hessian of $\Phi$ along $R^{i}(K) \times\{0\}$. Notice that $\Phi$ involves a sum of terms of the form $w \cdot w / 4$ where $w$ is a function from a Riemannian manifold into an inner product space, and the critical values occur on the locus $w=0$. Computing the derivative of a term of this form using the product rule we get $D(w \cdot w) / 4=$ $(1 / 2) D w \cdot w$. Differentiating again we get

$$
(1 / 2)\left(D^{2} w \cdot w+D w \cdot D w\right) .
$$

This handles the Hessian of the terms involving $\left\|\vec{w}_{i}\right\|^{2}$. The terms of the form $t^{2}\left\|\bar{v}_{i}\right\|^{2}$ are easier as we are only interested along the locus $t=0$. Hence the only contributions come from differentiating with respect to $t$ twice. 
Since we only care about the case when $w=0$, we get

$$
H(w)=(1 / 2) D w \cdot D w .
$$

Since the sum of the Hessians is the Hessian of the sum, we have that, along $R^{i}\left(S^{3}-\right.$ $N(K)) \times\{0\}$,

$$
H\left(\frac{\sum_{i}\left\|\vec{w}_{i}\right\|^{2}+t^{2}\left\|\bar{v}_{i}\right\|^{2}}{4}\right)=\frac{1}{2} \sum_{i}\left(\begin{array}{cc}
D \vec{w}_{i} \cdot D \vec{w}_{i} & 0 \\
0 & \bar{v}_{i} \cdot \bar{v}_{i}
\end{array}\right),
$$

where we split the tangent space of $\left(\operatorname{SU}(2)^{k}-(S \cup T)\right) \times \mathbb{R}$ at $(\rho, 0)$ as

$$
\mathrm{T}_{\rho} \mathrm{SU}(2)^{k} \oplus \mathrm{T}_{0} \mathbb{R} .
$$

From the properties of the logarithm, and the fact that we are working along the locus where the relators take on the value 1 , we have that $D \vec{w}_{i}=D r_{i}$ (compare with (32)). The Hessian splits as a direct sum over orthogonal matrices so that

$$
\sqrt{\operatorname{det}\left(H_{N}(\Phi)\right)}=\frac{1}{2^{(3 k-3) / 2}} \frac{\|d \bar{r}\|}{\|\vec{v}\|} .
$$

Assuming that the singularities of $\Phi$ are tame with respect to the Gaussian, we see that $S \cup T$ has measure zero. The functions $f$ are bounded, so we can consider the following limit:

$$
\lim _{\lambda \rightarrow \infty}\left(\frac{\lambda}{4 \pi}\right)^{(3 k-3) / 2} \int_{\mathrm{SU}(2)^{k} \times \mathbb{R}} f \exp \left(-\lambda \frac{\sum_{i}\left\|\vec{w}_{i}\right\|^{2}+t^{2}\left\|\bar{v}_{i}\right\|^{2}}{4}\right) v^{k} d \mathscr{L} .
$$

By Laplace's method this is equal to

$$
\left(\frac{1}{4}\right)^{(3 k-3) / 2} \int_{R^{i}\left(S^{3}-N(K)\right)} \frac{f v^{R}}{\sqrt{\operatorname{det}\left(H_{N}(\Phi)\right)}},
$$

where $v^{R}$ is the Riemannian metric on $R^{i}\left(S^{3}-N(K)\right)$ inherited from $\mathrm{SU}(2)^{k}$ and $\mid \operatorname{det}\left(H_{N}(\Phi) \mid\right.$ is the determinant of the Hessian of $\Phi$ restricted to the normal of the tangent space to $R^{i}\left(S^{3}-N(K)\right)$ in $\mathrm{SU}(2)^{k}$. Substituting the value for the Hessian computed in (43) this is further equal to

$$
\left(\frac{1}{4}\right)^{(3 k-3) / 2} 2^{(3 k-3) / 2} \int_{R^{i}\left(S^{3}-N(K)\right)} \frac{f\|\vec{v}\| \nu^{R}}{\|d \bar{r}\|}
$$

By (20) this is a multiple of the integral against Dubois torsion,

$$
\frac{1}{2^{(3 k-2) / 2}} \int_{R^{i}\left(S^{3}-N(K)\right)} f d \tau \text {. }
$$



form

Going back to (44), the dependence on $t$ can be factored out so that it is of the

$$
\begin{aligned}
& \left(\frac{\lambda}{4 \pi}\right)^{(3 k-3) / 2} \int_{\mathrm{SU}(2)^{k} \times \mathbb{R}} f \exp \left(-\lambda \frac{\sum_{i}\left\|\vec{w}_{i}\right\|^{2}+t^{2}\left\|\bar{v}_{i}\right\|^{2}}{4}\right) v^{k} d \mathscr{L} \\
& \quad=\left(\frac{\lambda}{4 \pi}\right)^{(3 k-3) / 2} \int_{\mathrm{SU}(2)^{k}} f \exp \left(-\lambda \frac{\sum_{i}\left\|\vec{w}_{i}\right\|^{2}}{4}\right)\left(\int_{\mathbb{R}} \exp \left(-\lambda \frac{t^{2}\|\vec{v}\|}{4}\right) d \mathscr{L}\right) v^{k} .
\end{aligned}
$$

Next we integrate out the $t$ to get,

$$
\sqrt{\frac{4 \pi}{\lambda}}\left(\frac{\lambda}{4 \pi}\right)^{(3 k-3) / 2} \int_{\mathrm{SU}(2)^{k}} f \exp \left(-\lambda \frac{\sum_{i}\left\|\vec{w}_{i}\right\|^{2}}{4}\right)\|\vec{v}\| v^{k} .
$$

Putting this into the equation derived from Laplace's method we have,

$$
\begin{aligned}
\lim _{\lambda \rightarrow \infty} & \sqrt{\frac{4 \pi}{\lambda}}\left(\frac{\lambda}{4 \pi}\right)^{(3 k-3) / 2} \int_{\mathrm{SU}(2)^{k}} f \exp \left(\lambda \frac{\sum_{i}\left\|\vec{w}_{i}\right\|^{2}}{4}\right)\|\vec{v}\| v^{k} \\
= & \frac{1}{2^{(3 k-2) / 2}} \int_{R^{i}\left(S^{3}-N(K)\right)} f d \tau .
\end{aligned}
$$

Finally, we recognize

$$
\left(\frac{\lambda}{4 \pi}\right)^{(3 k-3) / 2} \exp \left(-\lambda \frac{\sum_{i}\left\|\vec{w}_{i}\right\|^{2}}{4}\right)=\prod_{j} \beta_{\lambda}\left(r_{j}\right),
$$

which ends the proof of the theorem.

Since the Gaussian is pointwise close to the heat kernel trace we can replace $\beta_{\lambda}$ by $\kappa_{\lambda}$ in the right hand side of the equation (41) to get

$$
\lim _{\lambda \rightarrow \infty} \sqrt{\frac{4 \pi}{\lambda}} \int_{\mathrm{SU}(2)^{k}} 2^{\frac{3 k-3}{2}} f\|\vec{v}\| \prod_{i=1}^{k-1} \kappa_{\lambda}\left(r_{i}\right) \nu^{k},
$$

which yields a global formula for Dubois torsion.

Theorem 5.4 (Global formula). Let $K$ be a knot in $S^{3}$ such that the regular representations $R^{i}\left(S^{3}-N(K)\right)$ form an open dense subset of $R\left(S^{3}-N(K)\right)$ and the total Dubois torsion of the knot $K$ is finite, and suppose that the map $\Phi$ defined by (40) has tame singularities. For any smooth invariant function $f: \mathrm{SU}(2)^{k} \rightarrow \mathbb{R}$ we have

$$
\int_{R^{i}\left(S^{3}-N(K)\right)} f d \tau=\lim _{\lambda \rightarrow \infty} 2^{\frac{3 k-3}{2}} \sqrt{\frac{4 \pi}{\lambda}} \int_{\mathrm{SU}(2)^{k}} f\|\vec{v}\| \prod_{i=1}^{k-1} \kappa_{\lambda}\left(r_{i}\right) v^{k}
$$

Here $\kappa_{\lambda}$ is the Dirac delta function defined in Section 5.1, the maps $r_{j}$ are the relator maps, vector $\vec{v}$ is given by (12) and (37), $v^{k}$ is the volume form from the inclusion of $\mathrm{SU}(2)^{k}$ into $\mathbb{R}^{4 k}$, and $d \tau$ is the measure from Dubois torsion. 
Proof. This follows directly from Theorem 5.3 and Lemma 5.1.

\section{Interpreting the global formula}

The purpose of this section is to interpret integration against Dubois torsion in terms of quantum invariants of the knot complement.

Suppose that the assumptions of Theorem 5.4 are satisfied. Substituting (33) and (34) into the global formula (45) for integration against the Dubois torsion yields the following:

$$
\begin{aligned}
& \int_{R^{i}\left(S^{3}-N(K)\right)} f d \tau \\
& =\lim _{\lambda \rightarrow \infty} 2^{\frac{3 k-3}{2}} \sqrt{\frac{4 \pi}{\lambda}} \int_{\mathrm{SU}(2)^{k}} f\|\vec{v}\| \prod_{i=1}^{k-1} \frac{1}{2 \pi^{2}} \\
& \sum_{c=0}^{\infty}(-1)^{c}(c+1) e^{-c(c+2) / \lambda} s_{c}\left(-\operatorname{tr}\left(r_{i}\right)\right) \nu^{k} .
\end{aligned}
$$

In order to relate this formula to quantum invariants we need to recall the definition of the Yang-Mills measure [8].

6.1. The Yang-Mills measure in a handlebody. In this section we recall the definitions of the Kauffman bracket skein module of a handlebody and of the Yang-Mills measure functional on that module. A good reference for the standard definitions is the book [35]. More details and proofs relating to the Yang-Mills measure can be found in [8].

Given $t \in \mathbb{C}$, recall that the Kauffman bracket skein module of a manifold $M$, denoted by $K_{t}(M)$, is defined as a quotient of a vector space over $\mathbb{C}$, with basis given by the set of equivalence classes of framed links in the manifold (including an empty link), by the relations that define the Kauffman bracket:

and

$$
\bigcirc \cup L=-\left(t^{2}+t^{-2}\right) L
$$

$$
\left.\lambda=t \stackrel{\smile}{\curvearrowright}+t^{-1}\right)(.
$$

The elements of the Kauffman bracket skein module are called skeins.

Let $H$ be a handlebody. There is a convention of modeling skeins on admissibly colored framed trivalent graphs in $H$. An admissible coloring is an assignment of a nonnegative integer to each edge, so that the colors at trivalent vertices satisfy all possible triangle inequalities. The skein corresponding to such a graph is obtained by inserting an appropriate Jones-Wenzl idempotent at each edge, inserting a Kauffman 
triad at each vertex, and joining those with parallel strands. For the definitions of the Jones-Wenzl idempotents and the Kauffman triads see [35].

The Yang-Mills measure is a local, diffeomorphism invariant trace defined on $K_{t}(H)$,

$$
\mathrm{YM}: K_{t}(H) \longrightarrow \mathbb{C}
$$

Here is how to compute YM. Given a handlebody $H$ of genus $g$, its double is diffeomorphic to the connected sum of $g$ copies of $S^{1} \times S^{2}$. The Yang-Mills measure YM is the linear functional given by taking the inclusion of $H$ into $\sharp_{g} S^{1} \times S^{2}$ followed by the canonical isomorphism of $K_{t}\left(\sharp_{g} S^{1} \times S^{2}\right)$ with $\mathbb{C}$. This isomorphism can be described in the following way. Choose a system of spheres that cut down $\sharp_{g} S^{1} \times S^{2}$ to a punctured ball. Represent a skein as a linear combination of colored trivalent graphs intersecting the spheres transversely in the interior of edges, with each graph intersecting any sphere at most once. If a graph intersects a nonseparating sphere in a single point on one edge then this graph represents zero in the skein module. Thus we can assume that the graphs miss the spheres. The Yang-Mills measure is the value of the Kauffman bracket of the resulting skein in the punctured ball.

Alternatively, a handlebody $H$ can be thought of as $H=F \times I$, where $F$ is a compact, oriented surface with boundary. The skein module of $F \times I$ has an algebra structure, where multiplication is generated by laying one skein over another, with the direction given by the interval $I$. Choosing a trivalent spine for $F$ yields a basis for $K_{t}(H)$ given by the skeins corresponding to all possible admissible colorings of that spine. The Yang-Mills measure of any skein is the coefficient of the skein coming from labeling all the edges of the spine with 0 . Note that this does not apply to the skein algebra of the disk (which is spanned by the empty skein) nor to the skein module of the annulus (i.e. of the solid torus), which is spanned by all nonnegative colorings of the core of the annulus.

When $t=e^{\frac{\pi i}{2 r}}$, the Yang-Mills measure is defined the same way as above on the reduced Kauffman bracket skein module, $K_{r, f}(H)$, which is obtained from $K_{t}(H)$ by taking its quotient by the submodule spanned by all the skeins corresponding to the framed trivalent graphs where some edge is colored with $r-1$. The canonical basis for $K_{r, f}(H)$ is finite since colors cannot be larger than $r-2$. In this case the admissibility condition carries an additional requirement that the sum of the three colors at any vertex is less than or equal to $2 r-4$.

The projector $\omega$ is an element of the reduced skein module of the solid torus defined below:

$$
\omega=\sum_{i=0}^{r-2}(-1)^{i}[i+1] s_{i},
$$

where $[n]$ denotes the quantum integer,

$$
[n]=\frac{t^{2 n}-t^{-2 n}}{t^{2}-t^{-2}}
$$


and $s_{i}$ denotes the skein in the annulus which is the result of coloring the core with the $i$-th Jones-Wenzl idempotent. Note that $[n]$ is defined for all $t \neq 0$. At $t= \pm 1$ the formula we gave has a removable singularity and $\lim _{t \rightarrow \pm 1}[n]=n$. As the level $r$ approaches infinity, $t$ approaches -1 .

Evaluating the Yang-Mills measure of a skein $s$ in the handlebody $H$ can be understood in terms of the Kauffman bracket of a surgery diagram in the three-sphere. Embed $H$ in $S^{3}$ so that its complement is also a handlebody. Put an unknotted zeroframed circle decorated with the projector $\omega$ around each handle of $H$, and then take the value of the Kauffman bracket in the 3-ball of the skein which is a union of those decorated circles with the skein $s$. Finally divide the result by $X^{2 g}$, where

$$
X=\sqrt{\sum_{c=0}^{r-2}[c+1]^{2}} .
$$

The fact that this agrees with the definition of the Yang-Mills measure follows from the Turaev-Wenzl identity, fusion, and the shadow world formula for the Yang-Mills measure [23].

When the parameter $t$ is equal to -1 , the algebra $K_{-1}(H)$ is isomorphic to the coordinate ring of the $\mathrm{SU}(2)$-characters of $\pi_{1}(H)$; see [6] and [39]. The isomorphism is given by sending a skein given by a disjoint union of simple closed curves $c_{i}$ to the function that sends a representation $\rho$ to

$$
\prod_{i}-\operatorname{tr}\left(\rho\left(c_{i}\right)\right)
$$

Weyl orthogonality implies that via this isomorphism at $t=-1$ the Yang-Mills measure on a handlebody of genus $g$ is equal to integration on $\mathrm{SU}(2)^{g}$ against Haar measure.

6.2. Quantum 3-manifold invariants. In this section we discuss the evaluation of some quantum invariants of 3-manifolds.

Suppose that a closed, oriented 3-manifold $M$ is obtained by surgery on a framed link $L$. Let $b_{+}$(respectively $b_{-}$) denote the number of positive (negative) eigenvalues of the linking matrix of $L$. The Witten-Reshetikhin-Turaev invariant of $M$ at level $r$, where $r>3$ is a positive integer, can be obtained as the following expression:

$$
Z_{r}(M)=\langle L(\omega)\rangle\left\langle U_{+}(\omega)\right\rangle^{-b_{+}}\left\langle U_{-}(\omega)\right\rangle^{-b_{-}} .
$$

In this formula $L(\omega)$ denotes decorating each component of a given link with $\omega$, the symbol $U_{+}$(respectively $U_{-}$) denotes an unknot with framing +1 (respectively -1 ), and $\langle L\rangle$ denotes taking the value of the Kauffman bracket of a link $L$ in a 3-sphere evaluated at $4 r$-th root of unity. This formula is taken from Lickorish [35]. Note that in this normalization, $Z_{r}\left(S^{3}\right)=1$ for all $r$. 
The Turaev-Viro invariant, TV $(M)$, was originally defined combinatorially in [47] using triangulations of 3-manifolds and quantum $6 j$-symbols. Roberts [41] proved that the square of the norm of the Witten-Reshetikhin-Turaev invariant of any 3-manifold $M$ is equal to TV $(M)$. The proof involved showing that the Turaev-Viro invariant is equal to the chain-mail invariant derived from a Heegaard diagram of $M$. Roberts used a different normalization than Lickorish. His normalization agrees with Witten's, where $Z_{r}\left(S^{3}\right)=\frac{1}{X}$.

Consider the presentation of the 3-manifold $M$ by a Heegaard diagram on a standard handlebody $H$ in $S^{3}$. Let $\Omega=\frac{1}{X} \omega$. The chain-mail link consists of the attaching curves decorated with $\Omega$ along with the push-offs of a complete set of meridians of $H$, which link the attaching curves and are the 0 -framed unlink with $g$ components, and are also decorated with $\Omega$. The chain-mail invariant is equal to the value of the Kauffman bracket of the chain-mail link multiplied by $\frac{1}{X^{2}}$. Note that the link consisting of meridians and attaching curves obtained from a Heegaard diagrams yields a surgery presentation for $M \sharp \bar{M}$.

While the Yang-Mills measure is defined at all roots of unity (including $t=-1$ ) and when the complex parameter $t$ has absolute value different than 1, the WittenReshetikhin-Turaev and Turaev-Viro invariants of 3-manifolds are not defined away from roots of unity nor for $t=-1$. Although the terms of the state sum originally used to define the Turaev-Viro invariant of a manifold $M$ are all well defined when the value of the parameter is equal to -1 , the sum is infinite and does not converge.

6.3. Total torsion versus quantum invariants. By (46) the total Dubois torsion is equal to

$$
\lim _{\lambda \rightarrow \infty} 2^{\frac{3 k-3}{2}} \sqrt{\frac{4 \pi}{\lambda}} \int_{\mathrm{SU}(2)^{k}}\|\vec{v}\| \prod_{i=1}^{k-1} \frac{1}{2 \pi^{2}} \sum_{c=0}^{\infty}(-1)^{c}(c+1) e^{-c(c+2) / \lambda} S_{c}\left(-\operatorname{tr}\left(r_{i}\right)\right) \nu^{k} .
$$

Recall from Section 6.1 that integration on $\mathrm{SU}(2)^{k}$ against Haar measure agrees with the Yang-Mills measure on the Kauffman bracket skein module of a handlebody of genus $k$ at $t=-1$. Note also that the induced Riemannian measure on $\mathrm{SU}(2)$ viewed as $S^{3} \subset \mathbb{R}^{4}$ is equal to $\pi^{2}$ times the Haar measure. Unfortunately, it is difficult to recognize the integrand in (49) as a skein. Looking at a portion of the formula (which is just the Fourier expansion of the Dirac delta over SU(2) with respect to Haar measure)

$$
\sum_{c=0}^{\infty}(-1)^{c}(c+1) s_{c}\left(-\operatorname{tr}\left(r_{i}\right)\right)=\lim _{n \rightarrow \infty} \sum_{c=0}^{n}(-1)^{c}(c+1) s_{c}\left(-\operatorname{tr}\left(r_{i}\right)\right),
$$

and comparing it with (47), note that on the right we have a skein in a handlebody, which is obtained by decorating the attaching curve for a handle corresponding to the $i$-th relator with an analogue of $\omega \in K_{r, f}\left(S^{1} \times D^{2}\right)$. Thus we can view the left-hand side of (50) as coloring the attaching curves corresponding to relators $r_{i}$ 
with $\omega_{\infty}$. Recall now the method of computing the Yang-Mills measure in $K_{r, f}$ via the Kauffman bracket of a surgery diagram in the 3-sphere. The analogous equation in $K_{r, f}$ for the value of the Yang-Mills measure of the skein

$$
\prod_{i=1}^{k-1} \sum_{c=0}^{r-1}(-1)^{c}(c+1) s_{c}\left(-\operatorname{tr}\left(r_{i}\right)\right)
$$

is obtained by computing the Kauffman bracket of a chain mail link, which is a surgery diagram for the double of the knot complement.

If we tried naively to extend the Witten-Reshetikhin-Turaev invariant from $4 r$-th roots of unity to the value $t=-1$, we could begin by trying to compute the Kauffman bracket of a chain mail link, where instead of decorating the appropriate curves with $\omega$ we used $\omega_{\infty}$. Unfortunately this infinite sum does not converge. The corresponding model, called the Ponzano-Regge model, was studied in [1], [3], [18], and [19], where one can find explicit conditions guaranteeing convergence, divergence and invariance.

The second attempt would be to regularize using a bump function. Thus we could interpret the limit

$$
\lim _{\lambda \rightarrow \infty} \sqrt{\frac{4 \pi}{\lambda}} \int_{\mathrm{SU}(2)^{k}} \prod_{i=1}^{k-1} \frac{1}{2 \pi^{2}} \sum_{c=0}^{\infty}(-1)^{c}(c+1) e^{-c(c+2) / \lambda} s_{c}\left(-\operatorname{tr}\left(r_{i}\right)\right) \nu^{k}
$$

as the regularized Witten-Reshetikhin-Turaev invariant of the double of the knot complement at level -1 . Alas, this quantity is not a manifold invariant. In order to obtain an invariant we need to multiply the integrand by the factor $\|\vec{v}\|$, where $\vec{v}$ is the vector (defined by (12)), which appears in the computation of the Dubois torsion. Appearance of this vector was interpreted in Remark 4.4. This way we get an invariant of the double of the knot complement, which is equal to the total Dubois torsion of the knot.

The limit of the Witten-Reshetikhin-Turaev invariants of the double of the knot complement, as the level $r$ tends to infinity, does not exist due to oscillation. However, the deliberations in this paper lead us to the following conjecture.

Conjecture 6.1. Let $K$ be a knot in $S^{3}$ whose complement has sufficiently regular $\mathrm{SU}(2)$-representation variety. Denote by $T^{2}$ the boundary torus of the knot complement and by $M$ the 3-manifold which is the double of the knot complement. Let $f \in K_{-1}\left(T^{2}\right)$ be a peripheral skein, and let $Z_{r}(M)$ denote the Witten-ReshetikhinTuraev invariant of $M$ at level $r$. Then

$$
\lim \sup _{r \rightarrow \infty} \frac{1}{r}\left|Z_{r}(M, f)\right|
$$

defines a seminorm on $K_{-1}(T)$ whose radical is the A-ideal of the knot. 
Recall the conjectured formula

$$
\begin{aligned}
& Z_{r}^{\mathrm{SU}(2)}(M) \\
& \quad \sim_{r \rightarrow \infty} e^{-3 \pi \mathbf{i}\left(1+b^{1}(M)\right) / 4} \\
& \quad \times \int_{[A]} e^{2 \pi \mathbf{i} \mathrm{CS}(A)} r^{\left(h_{A}^{1}-h_{A}^{0}\right) / 2} e^{-2 \pi \mathbf{i}\left(I_{A} / 4+h_{A}^{0} / 8\right)} \tau_{M}(A)^{1 / 2}
\end{aligned}
$$

for the leading asymptotics of the Witten-Reshetikhin-Turaev invariant. Let $M$ be the double of the knot complement $S^{3}-N(K)$. We consider the right hand side of (51) at characters of representations of $M$ that come from bending regular representations of $\pi_{1}\left(S^{3}-N(K)\right)$. With this restriction $h^{1}=2, h^{0}=0, b^{1}(M)=1$, the ChernSimons invariant is an integer, and the spectral flow is zero; see [31] and [32]. Thus along the regular representations the absolute value of the right hand side of (51) reduces to

$$
r \int_{[A] \in X^{i}(M)} \tau_{M}(A)^{1 / 2}
$$

If we naively assume that there were no contributions to the leading order asymptotics coming from the singular points of the character variety, then the total Dubois torsion of $S^{3}-N(K)$ is proportional to the leading order asymptotics of the WittenReshetikhin-Turaev invariants of the double.

Comparing this now to Conjecture 6.1, we are working with the conjectural asymptotic formula extended to the Witten-Reshetikhin-Turaev invariant for a knot that lies in the boundary of the knot complement inside of the double of the knot complement. In the case of knots with very well behaved character varieties our conjecture is a consequence of the conjectured asymptotics of the Witten-Reshetikhin-Turaev invariant.

\section{References}

[1] J. W. Barrett and I. Naish-Guzman, The Ponzano-Regge model. Classical Quantum Gravity 26 (2009), Article ID 155014. Zbl 1172.83017

[2] N. Bleistein and R. Handelsman, Asymptotic expansions of integrals. Second ed. Dover Publications, New York, 1986. MR 0863284 Zbl 0327.41027 (first ed.)

[3] V. Bonzom and M. Smerlak, Bubble divergences from twisted cohomology. Comm. Math. Phys. 312 (2012), 399-426. MR 2917170 Zbl 06117942

[4] E. Buffenoir and Ph. Roche, Two-dimensional lattice gauge theory based on a quantum group. Comm. Math. Phys. 170 (1995), 669-698. MR 1337137 Zbl 0843.17030

[5] D. Bullock, Estimating a skein module with $\mathrm{SL}_{2}(\mathbb{C})$ characters. Proc. Amer. Math. Soc. 125 (1997), 1835-1839. MR 1403115 Zbl 0866.57005 
[6] D. Bullock, Rings of $\mathrm{SL}_{2}(C)$-characters and the Kauffman bracket skein module. Comment. Math. Helv. 72 (1997), 521-542. MR 1600138 Zbl 0907.57010

[7] D. Bullock, C. D. Frohman, and J. Kania-Bartoszynska, Understanding the Kauffman bracket skein module. J. Knot Theory Ramifications 8 (1999), 265-277. MR 1691437 Zbl 0932.57015

[8] D. Bullock, C. D. Frohman, and J. Kania-Bartoszynska, The Yang-Mills measure in the Kauffman bracket skein module. Comment. Math. Helv. 78 (2003), 1-17. MR 1966749 Zbl 1031.57012

[9] G. Burde, SU(2)-representation spaces for two-bridge knot groups. Math. Ann. 288 (1990), 103-119. MR 1070927 Zbl 0694.57003

[10] D. Cooper, M. Culler, H. Gillet, D. D. Long, and P. B. Shalen, Plane curves associated to character varieties of 3-manifolds. Invent. Math. 118 (1994), 47-84. MR 1288467 Zbl 0842.57013

[11] R. H. Crowell and R. H. Fox, Introduction to knot theory. Based upon lectures given at Haverford College under the Philips Lecture Program. Ginn and Co., Boston etc., 1963. Reprint. Graduate Texts in Mathematics 57, Springer-Verlag, New York etc, 1977. MR 0146828 (original ed.) MR 0445489 (reprint) Zbl 0126.39105 (original ed.) Zbl 0362.55001 (reprint)

[12] G. De Rham, Introduction aux polynômes d'un nœd. Enseignment Math. 13 (1967), 187-192. MR 0240804 Zbl 0157.54803

[13] J. Dubois, A volume form on the SU(2)-representation space of knot groups, Algebr. Geom. Topol. 6 (2006), 373-404. MR 2220682 Zbl 1095.57012

[14] J. Dubois, Non abelian Reidemeister torsion and volume form on the SU(2)-representation variety of knot groups. Ann. Inst. Fourier (Grenoble) 55 (2005), 1685-1734. MR 2172277 Zbl 1077.57009

[15] J. Dubois and S. Garoufalidis, Rationality of the SL(2, $\mathbb{C})$-Reidemeister torsion in dimension 3. Preprint 2008. arXiv:0908.1690

[16] J. Dubois and R. Kashaev, On the asymptotic expansion of the colored Jones polynomial for torus knots. Math. Ann. 339 (2007), 757-782. MR 2341899 Zbl 1129.57015

[17] D. S. Freed, Reidemeister torsion, spectral sequences, and Brieskorn spheres. J. Reine Angew. Math. 429 (1992), 75-89. MR 1173116 Zbl 0743.57015

[18] L. Freidel and D. Louapre, Ponzano-Regge model revisited I. Gauge fixing, observables and interacting spinning particles. Classical Quantum Gravity 21 (2004), 5685-5726. MR 2107334 Zbl 1060.83013

[19] L. Freidel and D. Louapre, Ponzano-Regge model revisited II. Equivalence with ChernSimons. Preprint 2010. arXiv:gr-qc/0410141v3

[20] D. S. Freed and R. E. Gompf, Computer calculation of Witten's 3-manifold invariant. Comm. Math. Phys. 141 (1991), 79-117. MR 1133261 Zbl 0739.53065

[21] C. D. Frohman and R. Gelca, Skein modules and the noncommutative torus. Trans. Amer. Math. Sociologist. 352 (2000), 4877-4888. MR 1675190 Zbl 0951.57007

[22] C. D. Frohman, R. Gelca, and W. Lofaro, The A-polynomial from the noncommutative viewpoint. Trans. Amer. Math. Soc. 354 (2002), 735-747. MR 1862565 Zbl 0980.57002 
[23] C. D. Frohman and J. Kania-Bartoszynska, Shadow world evaluation of the Yang-Mills measure. Algebr. Geom. Topol. 4 (2004), 311-332. MR 2077668 Zbl 1063.57013

[24] C. D. Frohman and E. P. Klassen, Deforming representations of knot groups in SU(2). Comment. Math. Helv. 66 (1991), 340-361. MR 1120651 Zbl 0738.57001

[25] S. Garoufalidis and T. Q. Le, The colored Jones function is $q$-holonomic. Geom. Topol. 9 (2005), 1253-1293. MR 2174266 Zbl 1078.57012

[26] R. Gelca, Non-commutative trigonometry and the A-polynomial of the trefoil knot. Math. Proc. Cambridge Philos. Soc. 133 (2002), 311-323. MR 1912404 Zbl 1017.57002

[27] L. C. Jeffrey, Chern-Simons-Witten invariants of lens spaces and torus bundles, and the semiclassical approximation. Comm. Math. Phys. (3) 147 (1992), 563-604. MR 1175494 Zbl 0755.53054

[28] L. C. Jeffrey and J. Weitsman, Geometric quantization and Witten's semiclassical manifold invariants. In H. Osborn (ed.), Low-dimensional topology and quantum field theory. Proceedings of the NATO Advanced Research Workshop held in Cambridge, September 6-12, 1992. NATO Advanced Science Institutes Series B: Physics 315. Plenum Press, New York, 1993, 317-322. MR 1263995 Zbl 0928.57017

[29] L. C. Jeffrey and J. Weitsman, Half density quantization of the moduli space of flat connections and Witten's semiclassical manifold invariants. Topology 32 (1993), 509-529. MR 1231958 Zbl 0811.57017

[30] D. Johnson, A geometric form of Casson's invariant. Handwritten Notes. 1984.

[31] P. Kirk and E. Klassen, Chern-Simons invariants of 3-manifolds decomposed along tori and the circle bundle over the representation space of $T^{2}$. Comm. Math. Phys. 153 (1993), 521-557. MR 1218931 Zbl 0789.57011

[32] P. Kirk and M. Lesch, The $\eta$-invariant, Maslov index, and spectral flow for Dirac-type operators on manifolds with boundary. Forum Math. 16 (2004), 553-629. MR 2044028 Zbl 1082.58021

[33] E. Klassen, Representations of knot groups in SU(2). Trans. Amer. Math. Soc. 326 (1991), 795-828. MR 1008696 Zbl 0743.57003

[34] A. Lubotzky and A. R. Magid, Varieties of representations of finitely generated groups. Mem. Amer. Math. Soc. 58 (1985), 1-117. MR 0818915 Zbl 0598.14042

[35] W. B. R. Lickorish, An introduction to knot theory. Graduate Texts in Mathematics 175. Springer Verlag, New York, 1997. MR 1472978 Zbl 0886.57001

[36] A. A. Migdal, Recursion equations in gauge field theories. Zh. Eksp. Teor. Fiz. 69 (1975), 810-822. www.jetp.ac.ru/cgi-bin/dn/e_042_03_0413.pdf

[37] J. Milnor, Whitehead torsion. Bull. Amer. Math. Soc. 72 (1966), 358-426. MR 0196736 Zbl 0147.23104

[38] T. Ohtsuki, Problems on invariants of knots and 3-manifolds. In T. Ohtsuki, T. Kohno, T. Le, J. Murakami, J. Roberts, and V. Turaev (eds.), Invariants of knots and 3-manifolds. Proceedings of the workshop, Kyoto, Japan, September 17-21, 2001. Geometry and Topology Monographs 4. Geometry and Topology Publications, Coventry, 2002, 377-572. MR 2065029 Zbl 0996.00037

[39] J. H. Przytycki and A. S. Sikora, On skein algebras and $\mathrm{Sl}_{2}(\mathbb{C})$-character varieties. Topology 39 (2000), 115-148. MR 1710996 Zbl 0958.57011 
[40] D. B. Ray and I. M. Singer, R-torsion and the Laplacian on Riemannian manifolds. $A d v$. in Math. 7 (1971), 145-210. MR 0295381 Zbl 0239.58014

[41] J. Roberts, Skein Theory and Turaev-Viro Invariants. Topology 34 (1995). 771-789. MR 1362787 Zbl 0866.57014

[42] S. Rosenberg, The Laplacian on a Riemannian manifold. An introduction to analysis on manifolds. London Mathematical Society Student Texts 31. Cambridge University Press, Cambridge, 1997. MR 1462892 Zbl 0868.58074

[43] L. Rozansky, A large $k$ asymptotics of Witten's invariant of Seifert manifolds. Comm. Math. Phys. 171 (1995), 279-322. MR 0837.57014 Zbl 1344728

[44] E. H. Spanier, Algebraic topology. McGraw-Hill Book Co., New York etc., 1966. MR 0210112 Zbl 0145.43303

[45] V. G. Turaev, Torsions of 3-dimensional manifolds. Progress in Mathematics 208. Birkhäuser Verlag, Basel, 2002. MR 1958479 Zbl 1012.57002

[46] V. G. Turaev, Introduction to combinatorial torsions. Notes taken by Felix Schlenk. Lectures in Mathematics ETH Zürich, Birkhäuser Verlag, Basel, 2001. MR 1809561 Zbl 0970.57001

[47] V. G. Turaev and O. Y. Viro, State sum invariants of 3-manifolds and quantum $6 j$-symbols. Topology 31 (1992), 865-902. MR 1191386 Zbl 0779.57009

[48] A. Weil, Remarks on the cohomology of groups. Ann. of Math. (2) 80 (1964), 149-157. MR 0169956 Zbl 0192.12802

[49] E. Witten, On quantum gauge theories in two dimensions. Comm. Math. Phys.141 (1991), 153-209. MR 1133264 Zbl 0762.53063

[50] E. Witten, Quantum field theory and the Jones polynomial. Comm. Math. Phys. 121 (1989), 351-399. MR 0990772 Zbl 0667.57005

[51] R. Wong, Asymptotic approximations of integrals. Corrected reprint of the 1989 original. Classics in Applied Mathematics 34. Society for Industrial and Applied Mathematics (SIAM), Philadelphia, PA, 2001. MR 1851050 Zbl 1078.41001

Received January 14, 2011

Charles D. Frohman, Department of Mathematics, The University of Iowa, Iowa City, IA 52242, U.S.A.

E-mail: charles-frohman@uiowa.edu

Joanna Kania-Bartoszynska, Division of Mathematical Sciences,

National Science Foundation, Arlington, VA 22230, U.S.A.

E-mail: jkaniaba@nsf.gov 